\title{
Highly Enantioselective 1,3-Dipolar Cycloaddition Reactions of 2-Benzopyrylium-4-olate Catalyzed by Chiral Lewis Acids
}

\author{
Hiroyuki Suga, * Kei Inoue, Shuichi Inoue, and Akikazu Kakehi \\ Department of Chemistry and Material Engineering, Faculty of Engineering, Shinshu University, \\ Wakasato, Nagano 380-8553, Japan \\ sugahio@gipwc.shinshu-u.ac.jp
}

\section{Experimental Section}

General. Melting points were determined on a Yanaco MP-13 melting point apparatus and are uncorrected. IR spectra were taken with a JASCO FT/IR-5300S spectrophotometer. ${ }^{1} \mathrm{H}$ NMR spectra were recorded on a JEOL JNM-LA400 $(400 \mathrm{MHz})$ spectrometer. Chemical shifts are expressed in parts per million downfield from tetramethylsilane as an internal standard. ${ }^{13} \mathrm{C}$ NMR spectra were recorded on JEOL JNM-LA400 (100 MHz) spectrometer using broadband proton decoupling. Chemical shifts are expressed in parts per million downfield from tetramethylsilane, using the middle resonance of $\mathrm{CDCl}_{3}$ $(77.0 \mathrm{ppm})$ as an internal standard. High-resolution mass spectra were obtained on HITACHI M-80B spectrometer. Elemental analyses were performed on a Perkin-Elmer $2400 \mathrm{CHN}$ recorder. High performance liquid chromatography was performed on a SHIMADZU LC-VP system. Optical rotations were recorded with a JASCO P-1010 polarimeter. For preparative column chromatography, Wakogel C-300HG was employed. Medium-pressure liquid chromatography was carried out using a column packed with Wakogel C-300HG. All reactions were carried out under an argon atmosphere in dried glassware.

Materials. $o$-(Methoxycarbonyl)- $\alpha$-diazoacetophenone (1) was prepared by the procedure in the previous paper. ${ }^{1}$ Benzyloxyacetaldehyde (3) and its derivatives $\mathbf{4}-\mathbf{8}$ were prepared according to the procedure in the literatures. ${ }^{2}$ Benzyl pyruvate (10) was prepared by the procedure in the literature. ${ }^{3} 3$ Acryloyl-2-oxazolizinone (11) was prepared according to the procedure in the literature. ${ }^{4}$ Methyl pyruvate (9), $\mathrm{Rh}_{2}(\mathrm{OAc})_{4}, \mathrm{Sc}(\mathrm{OTf})_{3}$ and $\mathrm{Yb}(\mathrm{OTf})_{3}$ were commercially available, and used without further purification. 2,6-Bis(oxazolinyl)pyridines were prepared by the procedure in the literatures. ${ }^{5}$ Powdered $4 \AA$ molecular sieves was commercially available and dried by in vacuo at $250{ }^{\circ} \mathrm{C}$ for $12 \mathrm{~h}$ before use. $\mathrm{CH}_{2} \mathrm{Cl}_{2}$ was purified by distillation first from $\mathrm{CaCl}_{2}$ and then $\mathrm{CaH}_{2}$ under argon.

General Procedure for the Reaction of $\alpha$-Diazoacetophenone 1 with Benzyloxyacetaldehyde (3) or its Derivatives 4 - 8: A solution of 2,6-bis[(4S)-(-)-4-isopropyl-2-oxazolin-2yl]pyridine $((S, S)$-Pybox-i-Pr, $15.1 \mathrm{mg}, 0.05 \mathrm{mmol})$ in $\mathrm{CH}_{2} \mathrm{Cl}_{2}(1.5 \mathrm{~mL})$ was added to a suspension of $\mathrm{Sc}(\mathrm{OTf})_{3}(24.6 \mathrm{mg}, 0.05 \mathrm{mmol})$ and powdered $4 \AA$ molecular sieves $(\mathrm{MS} 4 \mathrm{~A}, 0.50 \mathrm{~g})$ in $\mathrm{CH}_{2} \mathrm{Cl}_{2}(1.0 \mathrm{~mL})$. After stirring the mixture for $2 \mathrm{~h}, \mathrm{Rh}_{2}(\mathrm{OAc})_{4}(4.4 \mathrm{mg}, 0.01 \mathrm{mmol})$ and a solution of benzyloxyacetaldehyde or its derivatives $(1.0 \mathrm{mmol})$ in $\mathrm{CH}_{2} \mathrm{Cl}_{2}(2.5 \mathrm{~mL})$ were successively added. The mixture was cooled to $-10{ }^{\circ} \mathrm{C}$, and then a solution of diazoacetophenone $1(102.1 \mathrm{mg}, 0.5 \mathrm{mmol})$ in $\mathrm{CH}_{2} \mathrm{Cl}_{2}(5 \mathrm{~mL})$ was added over a period of $1 \mathrm{~h}$. After removal of MS 4A through celite, the reaction 


\section{Supporting Information}

mixture was filtered through a plug of silica gel $(3 \mathrm{~cm})$ with AcOEt/hexane $(1: 1,80 \mathrm{~mL})$ as an eluent. The solvent was removed in vacuo to give the mixture, which was purified by medium pressure liquid chromatography (MPLC) (silica gel, 1:99 - 5:95 AcOEt/hexane).

7-Exo-(benzyloxy)methyl-5-methoxy-6,8-dioxabenzo[c]bicyclo[3.2.1]octan-2-one and 7Endo-(benzyloxy)methyl-5-methoxy-6,8-dioxabenzo[c]bicyclo[3.2.1]octan-2-one.

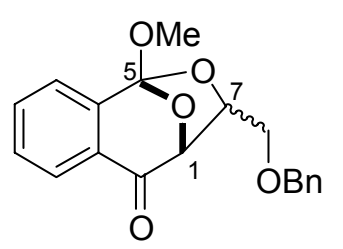

Benzyloxyaectaldehyde (3) $(150.2 \mathrm{mg}, 140 \mu \mathrm{L}, 1.0 \mathrm{mmol})$ was used according to the general procedure. Purification by MPLC (3:97 AcOEt/hexane) gave $18.7 \mathrm{mg}$ (11\%) of exo-cycloadduct and $139.2 \mathrm{mg}(85 \%)$ of endo-cycloadduct. Exocycloadduct: Colorless oil; IR (Neat) 2953, 2858, 1709, 1603, 1458, 1296, 1258, $1215,1161,1084,1046,951,764,748,700 \mathrm{~cm}^{-1} ;{ }^{1} \mathrm{H} \mathrm{NMR}\left(400 \mathrm{MHz}, \mathrm{CDCl}_{3}\right) \delta=$ $3.65(3 \mathrm{H}, \mathrm{s}), 3.69(1 \mathrm{H}, \mathrm{dd}, J=6.6,9.7 \mathrm{~Hz}), 3.75(1 \mathrm{H}, \mathrm{dd}, J=5.6,9.7 \mathrm{~Hz}), 4.04$ $(1 \mathrm{H}, \mathrm{dd}, J=5.6,6.6 \mathrm{~Hz}), 4.62(2 \mathrm{H}, \mathrm{s}), 4.82(1 \mathrm{H}, \mathrm{s}), 7.28-7.40(5 \mathrm{H}, \mathrm{m}), 7.45-7.53(1 \mathrm{H}, \mathrm{m}), 7.61-$ $7.67(2 \mathrm{H}, \mathrm{m}), 8.00(1 \mathrm{H}, \mathrm{d}, J=7.6 \mathrm{~Hz}) ;{ }^{13} \mathrm{C} \mathrm{NMR}\left(\mathrm{CDCl}_{3}, 100 \mathrm{MHz}\right) \delta=49.7,69.9,72.8,73.5,81.4$, 118.6, 122.6, 126.6, 127.7, 127.9, 128.2, 128.5, 129.3, 134.6, 137.6, 142.5, 192.9; HRMS (EI) Found: 326.1126. Calcd for $\mathrm{C}_{19} \mathrm{H}_{18} \mathrm{O}_{5}\left(\mathrm{M}^{+}\right): 326.1153 ; \mathrm{R}_{f}=0.42(2: 3 \mathrm{AcOEt} / \mathrm{hexane})$. Endo-cycloadduct: Colorless prisms; $\mathrm{mp}=67-69^{\circ} \mathrm{C} ;[\alpha]_{\mathrm{D}}{ }^{26}=-102^{\circ}\left(c 0.96, \mathrm{CHCl}_{3}\right)$; IR $(\mathrm{KBr}) 2957,2895,1711,1603$, 1456, 1379, 1318, 1287, 1248, 1217, 1157, 1090, 1049, 1026, 1007, 976, 947, 889, 752, 704, $594 \mathrm{~cm}^{-1}$; ${ }^{1} \mathrm{H}$ NMR $\left(\mathrm{CDCl}_{3}, 400 \mathrm{MHz}\right) \delta=3.30(1 \mathrm{H}, \mathrm{dd}, J=6.3,10.7 \mathrm{~Hz}), 3.46(1 \mathrm{H}, \mathrm{dd}, J=4.1,10.7 \mathrm{~Hz}), 3.68$ $(3 \mathrm{H}, \mathrm{s}), 4.32(1 \mathrm{H}, \mathrm{d}, J=12.0 \mathrm{~Hz}), 4.42(1 \mathrm{H}, \mathrm{d}, J=12.0 \mathrm{~Hz}), 4.60-4.65(1 \mathrm{H}, \mathrm{m}), 4.96(1 \mathrm{H}, \mathrm{d}, J=5.6$ $\mathrm{Hz}), 7.18-7.30(5 \mathrm{H}, \mathrm{m}), 7.41-7.44(1 \mathrm{H}, \mathrm{m}), 7.57-7.66(2 \mathrm{H}, \mathrm{m}), 7.92(1 \mathrm{H}, \mathrm{d}, J=7.8 \mathrm{~Hz}) ;{ }^{13} \mathrm{C} \mathrm{NMR}$ $\left(100 \mathrm{MHz}, \mathrm{CDCl}_{3}\right) \delta=49.6,68.0,73.5,74.8,81.7,118.1,122.6,126.2,127.7,127.8,128.3,129.1$, 129.3, 134.3, 137.3, 143.7, 192.4; HRMS (EI) Found: 326.1163. Calcd for $\mathrm{C}_{19} \mathrm{H}_{18} \mathrm{O}_{5}\left(\mathrm{M}^{+}\right): 326.1153$. $\mathrm{R}_{f}=0.35$ (2:3 AcOEt/hexane). The enantiomeric excess was determined by HPLC analysis (Daicel Chiralpak AS, 1:19 $i$-PrOH/hexane, Flow rate $=0.5 \mathrm{~mL} / \mathrm{min}, 35^{\circ} \mathrm{C}$ ). Exo-isomer: $\mathrm{t}_{\mathrm{R}}=13.7 \mathrm{~min}$ (minor) and 16.7 min (major). Endo-isomer: $\mathrm{t}_{\mathrm{R}}=12.3 \mathrm{~min}$ (minor) and $13.6 \mathrm{~min}$ (major).

5-Methoxy-7-exo-[(o-methoxybenzyl)oxy] methyl-6,8-dioxabenzo[c]bicyclo[3.2.1]octan-2one and 5-Methoxy-7-endo-[(o-methoxybenzyl)oxy] methyl-6,8-dioxabenzo[c]bicyclo[3.2.1] $]$ octan-2-

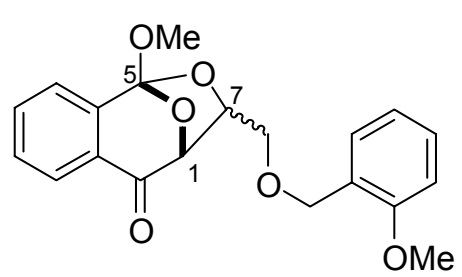

one. [(o-Methoxybenzyl)oxy]aectaldehyde (4) (164.2 $\mathrm{mg}, 1.0 \mathrm{mmol})$ was used according to the general procedure. The reaction mixture was quenched by addition of $\mathrm{NEt}_{3}(1.0 \mathrm{~mL})$ and stirred for additional $1 \mathrm{~h}$. Purification by MPLC (5:95 AcOEt/hexane) gave $21.4 \mathrm{mg}(12 \%)$ of exocycloadduct and $124.6 \mathrm{mg}(70 \%)$ of endo-cycloadduct. Exo-cycloadduct: Colorless prisms; $\mathrm{mp}=76-78.5^{\circ} \mathrm{C}$; IR (KBr) 3073, 3034, 3009, 2961, $2942,2909,2890,2876,2839,1711,1603,1591,1499,1483,1466,1441$, $1410,1373,1290,1273,1253,1213,1159,1140,1105,1088,1061,1049,1020,966,949 \mathrm{~cm}^{-1} ;{ }^{1} \mathrm{H}$ $\operatorname{NMR}\left(400 \mathrm{MHz}, \mathrm{CDCl}_{3}\right) \delta=3.66(3 \mathrm{H}, \mathrm{s}), 3.74(1 \mathrm{H}, \mathrm{dd}, J=6.9,9.9 \mathrm{~Hz}), 3.81(1 \mathrm{H}, \mathrm{dd}, J=5.4,9.9 \mathrm{~Hz})$, $3.84(3 \mathrm{H}, \mathrm{s}), 4.06(1 \mathrm{H}, \mathrm{dd}, J=5.4,6.9 \mathrm{~Hz}), 4.66(2 \mathrm{H}, \mathrm{s}), 4.85(1 \mathrm{H}, \mathrm{s}), 6.87-6.98(2 \mathrm{H}, \mathrm{m}), 7.26-7.39$ $(2 \mathrm{H}, \mathrm{m}), 7.46-7.50(1 \mathrm{H}, \mathrm{m}), 7.61-7.63(2 \mathrm{H}, \mathrm{m}), 8.00(1 \mathrm{H}, \mathrm{d}, J=7.8 \mathrm{~Hz})$; HRMS (EI) Found: 356.1254. Calcd for $\mathrm{C}_{20} \mathrm{H}_{20} \mathrm{O}_{6}\left(\mathrm{M}^{+}\right): 356.1259 ; \mathrm{R}_{f}=0.51$ (3:7 AcOEt/hexane). Endo-cycloadduct: Colorless prisms; $\mathrm{mp}=97.5-99.5{ }^{\circ} \mathrm{C} ;[\alpha]_{\mathrm{D}}{ }^{26}=-92^{\circ}\left(c\right.$ 0.96, $\left.\mathrm{CHCl}_{3}\right)$; IR $(\mathrm{KBr}) 3002,2959,2926$, 2895, 2870, 2843, 1705, 1603, 1495, 1462, 1441, 1373, 1314, 1294, 1244, 1227, 1159, 1136, 1190, $1076,1051,1028,974,953,943 \mathrm{~cm}^{-1} ;{ }^{1} \mathrm{H}$ NMR $\left(400 \mathrm{MHz}, \mathrm{CDCl}_{3}\right) \delta=3.32(1 \mathrm{H}, \mathrm{dd}, J=6.8,10.9 \mathrm{~Hz})$, $3.49(1 \mathrm{H}, \mathrm{dd}, J=4.1,10.9 \mathrm{~Hz}), 3.68(3 \mathrm{H}, \mathrm{s}), 3.76(3 \mathrm{H}, \mathrm{s}), 4.40(1 \mathrm{H}, \mathrm{d}, J=12.6 \mathrm{~Hz}), 4.48(1 \mathrm{H}, \mathrm{d}, J=$ $12.6 \mathrm{~Hz}), 4.63-4.67(1 \mathrm{H}, \mathrm{m}), 4.97(1 \mathrm{H}, \mathrm{d}, J=5.6 \mathrm{~Hz}), 6.80-6.89(2 \mathrm{H}, \mathrm{m}), 7.16-7.24(2 \mathrm{H}, \mathrm{m}), 7.42-$ $7.46(1 \mathrm{H}, \mathrm{m}), 7.58-7.66(2 \mathrm{H}, \mathrm{m}), 7.93(1 \mathrm{H}, \mathrm{d}, J=7.8 \mathrm{~Hz})$; HRMS (EI) Found: 356.1249. Calcd for $\mathrm{C}_{20} \mathrm{H}_{20} \mathrm{O}_{6}\left(\mathrm{M}^{+}\right): 356.1259 ; \mathrm{R}_{f}=0.45(3: 7 \mathrm{AcOEt} / \mathrm{hexane})$. The enantiomeric excess was determined by 


\section{Supporting Information}

HPLC analysis (Daicel Chiralpak AS, $1: 19 i$-PrOH/hexane, Flow rate $=0.5 \mathrm{~mL} / \mathrm{min}, 35{ }^{\circ} \mathrm{C}$ ). Exoisomer: $t_{\mathrm{R}}=13.2 \mathrm{~min}$ (minor) and $15.0 \mathrm{~min}$ (major). Endo-isomer: $\mathrm{t}_{\mathrm{R}}=13.7 \mathrm{~min}$ (minor) and $16.3 \mathrm{~min}$ (major).

5-Methoxy-7-exo-[(p-methoxybenzyl)oxy]methyl-5-methoxy-6,8-dioxabenzo[c]bicyclo[3.2.1]octan-2-one and 5-Methoxy-7-endo-[(p-methoxybenzyl)oxy]methyl-6,8-dioxabenzo[c]bicyclo[3.2.1]octan-2-one. [( $p$-Methoxybenzyl)oxy]aectaldehyde (5) $(164.2 \mathrm{mg}, 1.0 \mathrm{mmol})$ was used

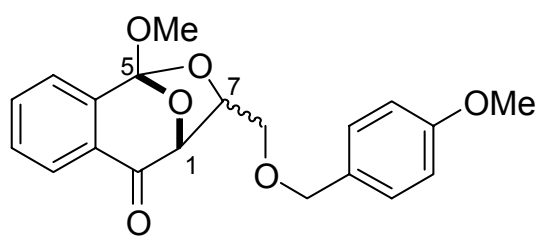
according to the general procedure. The reaction mixture was quenched by addition of $\mathrm{NEt}_{3}(1.0 \mathrm{~mL})$ and stirred for additional $1 \mathrm{~h}$. Purification by MPLC (5:95 AcOEt/hexane) gave $8.6 \mathrm{mg}(5 \%)$ of exo-cycloadduct and $85.5 \mathrm{mg}(48 \%)$ of endo-cycloadduct. Exocycloadduct: Colorless oil; IR (Neat) 2955, 2859, 1709, 1607, 1514, $1460,1294,1254,1215,1161,1101,1084,1065,1032,951 \mathrm{~cm}^{-1} ;{ }^{1} \mathrm{H}$ $\operatorname{NMR}\left(400 \mathrm{MHz}, \mathrm{CDCl}_{3}\right) \delta=3.64(3 \mathrm{H}, \mathrm{s}), 3.65(1 \mathrm{H}, \mathrm{dd}, J=6.8,9.8 \mathrm{~Hz}), 3.72(1 \mathrm{H}, \mathrm{dd}, J=5.5,9.8 \mathrm{~Hz})$, $3.81(3 \mathrm{H}, \mathrm{s}), 4.02(1 \mathrm{H}, \mathrm{dd}, J=5.5,6.8 \mathrm{~Hz}), 4.55(2 \mathrm{H}, \mathrm{s}), 4.80(1 \mathrm{H}, \mathrm{s}), 6.89(2 \mathrm{H}, \mathrm{d}, J=8.3 \mathrm{~Hz}), 7.27(2 \mathrm{H}$, $\mathrm{d}, J=8.3 \mathrm{~Hz}), 7.46-7.50(1 \mathrm{H}, \mathrm{m}), 7.61-7.63(2 \mathrm{H}, \mathrm{m}), 7.99(1 \mathrm{H}, \mathrm{d}, J=7.8 \mathrm{~Hz})$; HRMS (EI) Found: 356.1228. Calcd for $\mathrm{C}_{20} \mathrm{H}_{20} \mathrm{O}_{6}\left(\mathrm{M}^{+}\right): 356.1259 ; \mathrm{R}_{f}=0.48$ (3:7 AcOEt/hexane). Endo-cycloadduct: Colorless prisms; $\mathrm{mp}=75-77^{\circ} \mathrm{C} ;[\alpha]_{\mathrm{D}}{ }^{26}=-80^{\circ}\left(c 1.02, \mathrm{CHCl}_{3}\right)$; IR (KBr) 3034, 2994, 2955, 2924, 2863, 2849, 2834, 2807, 1705, 1605, 1588, 1512, 1468, 1458, 1441, 1414, 1372, 1314, 1294, 1244, $1223,1171,1155,1127,1103,1076,1057,1034,1013,976,951,889,841,816 \mathrm{~cm}^{-1}$; ${ }^{1} \mathrm{H}$ NMR $(400$ $\left.\mathrm{MHz}, \mathrm{CDCl}_{3}\right) \delta=3.26(1 \mathrm{H}, \mathrm{dd}, J=6.6,10.7 \mathrm{~Hz}), 3.41(1 \mathrm{H}, \mathrm{dd}, J=4.4,10.7 \mathrm{~Hz}), 3.68(3 \mathrm{H}, \mathrm{s}), 3.78(3 \mathrm{H}$, s), $4.25(1 \mathrm{H}, \mathrm{d}, J=11.5 \mathrm{~Hz}), 4.36(1 \mathrm{H}, \mathrm{d}, J=11.5 \mathrm{~Hz}), 4.59-4.63(1 \mathrm{H}, \mathrm{m}), 4.95(1 \mathrm{H}, \mathrm{d}, J=5.6 \mathrm{~Hz})$, $6.81(2 \mathrm{H}, \mathrm{d}, J=8.3 \mathrm{~Hz}), 7.13(2 \mathrm{H}, \mathrm{d}, J=8.3 \mathrm{~Hz}), 7.42-7.45(1 \mathrm{H}, \mathrm{m}), 7.58-7.66(2 \mathrm{H}, \mathrm{m}), 7.93(1 \mathrm{H}, \mathrm{d}$, $J=7.6 \mathrm{~Hz}$ ); HRMS (EI) Found: 356.1246. Calcd for $\mathrm{C}_{20} \mathrm{H}_{20} \mathrm{O}_{6}\left(\mathrm{M}^{+}\right): 356.1259 ; \mathrm{R}_{f}=0.42(3: 7$ AcOEt/hexane). The enantiomeric excess was determined by HPLC analysis. Exo-isomer (Daicel Chiralpak AS, 1:19 $i-\mathrm{PrOH} /$ hexane, Flow rate $=0.5 \mathrm{~mL} / \mathrm{min}, 35^{\circ} \mathrm{C}$ ): $\mathrm{t}_{\mathrm{R}}=27.9 \mathrm{~min}$ (major) and $42.5 \mathrm{~min}$ (minor). Endo-isomer (Daicel Chiralpak AD, 1:19 $i$-PrOH/hexane, Flow rate $=0.5 \mathrm{~mL} / \mathrm{min}, 35^{\circ} \mathrm{C}$ ): $t_{\mathrm{R}}$ $=24.5 \mathrm{~min}$ (minor) and $27.5 \mathrm{~min}$ (major).

7-Exo-[(p-fluorobenzyl)oxy]methyl-5-methoxy-6,8-dioxabenzo[c]bicyclo[3.2.1]octan-2-one and 7-Endo-[(p-fluorobenzyl)oxy]-5-methoxy-6,8-dioxabenzo[c]bicyclo[3.2.1]octan-2-one. $\quad[(p-$<smiles>CO[C@]1(COCc2ccc(F)cc2)O[C@]2(OC)C(=O)c3ccccc3C(=O)[C@]12I</smiles>
Fluorobenzyl)oxy]aectaldehyde (6) (168.2 $\mathrm{mg}, 1.0 \mathrm{mmol})$ was used according to the general procedure. Purification by MPLC (5:95 AcOEt/hexane) gave $29.4 \mathrm{mg}(17 \%)$ of exo-cycloadduct and $139.2 \mathrm{mg}$ (80\%) of endo-cycloadduct. Exo-cycloadduct: Colorless oil; IR (Neat) 2955, 2863, 1711, 1603, 1510, 1460, 1294, 1260, 1223, 1159, 1084, $1065,1046,1028,951,828 \mathrm{~cm}^{-1} ;{ }^{1} \mathrm{H}$ NMR $\left(400 \mathrm{MHz}, \mathrm{CDCl}_{3}\right) \delta=3.65$ $(3 \mathrm{H}, \mathrm{s}), 3.69(1 \mathrm{H}, \mathrm{dd}, J=6.6,9.6 \mathrm{~Hz}), 3.74(1 \mathrm{H}, \mathrm{dd}, J=5.6,9.6 \mathrm{~Hz}), 4.03(1 \mathrm{H}, \mathrm{dd}, J=5.6,6.6 \mathrm{~Hz})$, $4.58(2 \mathrm{H}, \mathrm{s}), 4.81(1 \mathrm{H}, \mathrm{s}), 6.99-7.07(2 \mathrm{H}, \mathrm{m}), 7.21-7.34(2 \mathrm{H}, \mathrm{m}), 7.47-7.51(1 \mathrm{H}, \mathrm{m}), 7.62-7.66$ $(2 \mathrm{H}, \mathrm{m}), 8.00(1 \mathrm{H}, \mathrm{d}, J=7.6 \mathrm{~Hz})$; HRMS (EI) Found: 344.1076. Calcd for $\mathrm{C}_{19} \mathrm{H}_{17} \mathrm{FO}_{5}\left(\mathrm{M}^{+}\right): 344.1059$; $\mathrm{R}_{f}=0.46(3: 7 \mathrm{AcOEt} /$ hexane $)$. Endo-cycloadduct: Colorless needles; $\mathrm{mp}=112-114{ }^{\circ} \mathrm{C} ;[\alpha]_{\mathrm{D}}{ }^{26}=-$ $98^{\circ}$ (c 1.00, $\mathrm{CHCl}_{3}$ ); IR (KBr) 2963, 2919, 2897, 2857, 1709, 1603, 1512, 1460, 1447, 1379, 1318, $1292,1248,1223,1155,1132,1092,1073,1049,1026,1007,974,947,891,858,839 \mathrm{~cm}^{-1}$; ${ }^{1} \mathrm{H}$ NMR $\left(400 \mathrm{MHz}, \mathrm{CDCl}_{3}\right) \delta=3.31(1 \mathrm{H}, \mathrm{dd}, J=6.3,10.7 \mathrm{~Hz}), 3.46(1 \mathrm{H}, \mathrm{dd}, J=4.4,10.7 \mathrm{~Hz}), 3.68(3 \mathrm{H}, \mathrm{s})$, $4.28(1 \mathrm{H}, \mathrm{d}, J=11.7 \mathrm{~Hz}), 4.36(1 \mathrm{H}, \mathrm{d}, J=11.7 \mathrm{~Hz}), 4.62(1 \mathrm{H}, \mathrm{m}), 4.96(1 \mathrm{H}, \mathrm{d}, J=5.6 \mathrm{~Hz}), 6.93-6.97$ 


\section{Supporting Information}

$(2 \mathrm{H}, \mathrm{m}), 7.13-7.17(2 \mathrm{H}, \mathrm{m}), 7.41-7.45(1 \mathrm{H}, \mathrm{m}), 7.58-7.66(2 \mathrm{H}, \mathrm{m}), 7.90(1 \mathrm{H}, \mathrm{d}, J=7.6 \mathrm{~Hz})$; HRMS (EI) Found: 344.1068. Calcd for $\mathrm{C}_{19} \mathrm{H}_{17} \mathrm{FO}_{5}\left(\mathrm{M}^{+}\right): 344.1059 ; \mathrm{R}_{f}=0.39$ (3:7 AcOEt/hexane). The enantiomeric excess was determined by HPLC analysis. Exo-isomer (Daicel Chiralpak AD, 1:19 $i$ $\mathrm{PrOH} /$ hexane, Flow rate $=0.5 \mathrm{~mL} / \mathrm{min}, 35^{\circ} \mathrm{C}$ ): $\mathrm{t}_{\mathrm{R}}=20.1 \mathrm{~min}$ (minor) and $25.1 \mathrm{~min}$ (major). Endoisomer (Daicel Chiralpak AS, 1:19 $i-\mathrm{PrOH} /$ hexane, Flow rate $=0.5 \mathrm{~mL} / \mathrm{min}, 35^{\circ} \mathrm{C}$ ): $\mathrm{t}_{\mathrm{R}}=14.2 \mathrm{~min}$ (minor) and 16.1 min (major).

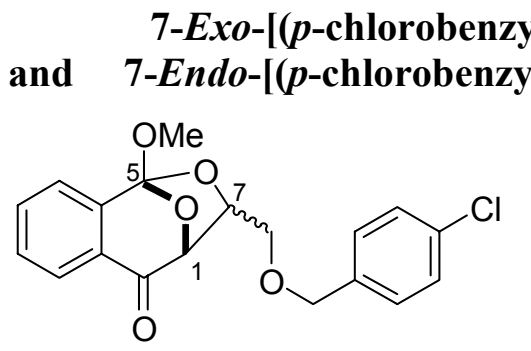

zyl)oxy]methyl-5-methoxy-6,8-dioxabenzo[c]bicyclo[3.2.1]octan-2-one oxy]methyl-5-methoxy-6,8-dioxabenzo[c]bicyclo[3.2.1]octan-2-one. [( $p$-Chlorobenzyl)oxy] acetaldehyde (7) $(184.6 \mathrm{mg}, 1.0 \mathrm{mmol})$ was used according to the general procedure. The reaction mixture was quenched by addition of $\mathrm{NEt}_{3}(1.0 \mathrm{~mL})$ and stirred for additional $1 \mathrm{~h}$. Purification by MPLC (5:95 AcOEt/hexane) gave $40.7 \mathrm{mg}(23 \%)$ of exocycloadduct and $111.5 \mathrm{mg}(62 \%)$ of endo-cycloadduct. Exocycloadduct: Colorless oil; IR (Neat) 2953, 2863, 1711, 1603, 1493, 1460, 1296, 1260, 1217, 1161, 1088, 1065, 1047, 1026, $951 \mathrm{~cm}^{-1} ;{ }^{1} \mathrm{H}$ NMR $\left(400 \mathrm{MHz}, \mathrm{CDCl}_{3}\right) \delta=3.65$ $(3 \mathrm{H}, \mathrm{s}), 3.69(1 \mathrm{H}, \mathrm{dd}, J=6.6,9.8 \mathrm{~Hz}), 3.74(1 \mathrm{H}, \mathrm{dd}, J=5.6,9.8 \mathrm{~Hz}), 4.04(1 \mathrm{H}, \mathrm{dd}, J=5.6,6.6 \mathrm{~Hz})$, $4.58(2 \mathrm{H}, \mathrm{s}), 4.81(1 \mathrm{H}, \mathrm{s}), 7.28(2 \mathrm{H}, \mathrm{d}, J=8.2 \mathrm{~Hz}), 7.33(2 \mathrm{H}, \mathrm{d}, J=8.2 \mathrm{~Hz}), 7.47-7.51(1 \mathrm{H}, \mathrm{m}), 7.62-$ $7.63(2 \mathrm{H}, \mathrm{m}), 8.00(1 \mathrm{H}, \mathrm{d}, J=7.6 \mathrm{~Hz})$; HRMS (EI) Found: 360.0782 . Calcd for $\mathrm{C}_{19} \mathrm{H}_{17} \mathrm{ClO}_{5}\left(\mathrm{M}^{+}\right)$: 360.0763; $\mathrm{R}_{f}=0.59$ (3: $7 \mathrm{AcOEt} /$ hexane). Endo-cycloadduct: Colorless needles; $\mathrm{mp}=103-106{ }^{\circ} \mathrm{C}$; $[\alpha]_{\mathrm{D}}{ }^{26}=-77^{\circ}\left(c 1.00, \mathrm{CHCl}_{3}\right)$; IR (KBr) 2957, 2897, 2855, 1709, 1601, 1495, 1476, 1458, 1408, 1381, 1316, 1292, 1248, 1219, 1155, 1134, 1090, 1073, 1049, 1017, 974, 945, $891 \mathrm{~cm}^{-1}$; ${ }^{1} \mathrm{H}$ NMR (400 MHz, $\left.\mathrm{CDCl}_{3}\right) \delta=3.31(1 \mathrm{H}, \mathrm{dd}, J=6.3,10.7 \mathrm{~Hz}), 3.46(1 \mathrm{H}, \mathrm{dd}, J=4.4,10.7 \mathrm{~Hz}), 3.68(3 \mathrm{H}, \mathrm{s}), 4.28(1 \mathrm{H}, \mathrm{d}, J$ $=12.0 \mathrm{~Hz}), 4.36(1 \mathrm{H}, \mathrm{d}, J=12.0 \mathrm{~Hz}), 4.59-4.64(1 \mathrm{H}, \mathrm{m}), 4.96(1 \mathrm{H}, \mathrm{d}, J=5.6 \mathrm{~Hz}), 7.10(2 \mathrm{H}, \mathrm{d}, J=8.3$ $\mathrm{Hz}), 7.23(2 \mathrm{H}, \mathrm{d}, J=8.3 \mathrm{~Hz}), 7.40-7.44(1 \mathrm{H}, \mathrm{m}), 7.58-7.66(2 \mathrm{H}, \mathrm{m}), 7.90(1 \mathrm{H}, \mathrm{d}, J=7.6 \mathrm{~Hz})$; HRMS (EI) Found: 360.0766. Calcd for $\mathrm{C}_{19} \mathrm{H}_{17} \mathrm{ClO}_{5}\left(\mathrm{M}^{+}\right): 360.0763 ; \mathrm{R}_{f}=0.53$ (3:7 AcOEt/hexane). The enantiomeric excess was determined by HPLC analysis (Daicel Chiralpak AS, 1:19 $i$-PrOH/hexane, Flow rate $=0.5 \mathrm{~mL} / \mathrm{min}, 35{ }^{\circ} \mathrm{C}$ ). Exo-isomer: $\mathrm{t}_{\mathrm{R}}=15.9 \mathrm{~min}$ (minor) and $18.8 \mathrm{~min}$ (major). Endoisomer: $\mathrm{t}_{\mathrm{R}}=13.7 \mathrm{~min}$ (minor) and $15.7 \mathrm{~min}$ (major).

7-Exo-[(p-bromobenzyl)oxy]methyl-5-methoxy-6,8-dioxabenzo[c]bicyclo[3.2.1]octan-2-one and 7-Endo-[(p-bromobenzyl)oxy]methyl-5-methoxy-6,8-dioxabenzo[c]bicyclo[3.2.1]octan-2-one.<smiles>CO[C@]1(COCc2ccc(Br)cc2)O[C@]2(OC)C(=O)c3ccccc3C(=O)[C@]12I</smiles>
[( $p$-Bromobenzyl)oxy]acetaldehyde $(\mathbf{8})(229.1 \mathrm{mg}, 1.0 \mathrm{mmol})$ was used according to the general procedure. The reaction mixture was quenched by addition of $\mathrm{NEt}_{3}(1.0 \mathrm{~mL})$ and stirred for additional $1 \mathrm{~h}$. Purification by MPLC (5:95 AcOEt/hexane) gave $56.8 \mathrm{mg}(28 \%)$ of exo-cycloadduct and $98.0 \mathrm{mg}(49 \%)$ of endo-cycloadduct. Exocycloadduct: Colorless oil; IR (Neat) 2953, 2859, 1713, 1603, 1487, 1460, 1294, 1260, 1217, 1161, 1084, 1046, $951 \mathrm{~cm}^{-1}$; ${ }^{1} \mathrm{H}$ NMR (400 MHz, $\left.\mathrm{CDCl}_{3}\right) \delta=3.65(3 \mathrm{H}, \mathrm{s}), 3.69$ $(1 \mathrm{H}, \mathrm{dd}, J=6.6,9.8 \mathrm{~Hz}), 3.74(1 \mathrm{H}, \mathrm{dd}, J=5.6,9.8 \mathrm{~Hz}), 4.03(1 \mathrm{H}, \mathrm{dd}, J=5.6,6.6 \mathrm{~Hz}), 4.57(2 \mathrm{H}, \mathrm{s})$, $4.81(1 \mathrm{H}, \mathrm{s}), 7.23(2 \mathrm{H}, \mathrm{d}, J=8.0 \mathrm{~Hz}), 7.48-7.50(3 \mathrm{H}, \mathrm{m}), 7.62-7.63(2 \mathrm{H}, \mathrm{m}), 8.00(1 \mathrm{H}, \mathrm{d}, J=7.6$ $\mathrm{Hz}$ ); HRMS (EI) Found: 404.0276. Calcd for $\mathrm{C}_{19} \mathrm{H}_{17} \mathrm{BrO}_{5}\left(\mathrm{M}^{+}\right)$: 404.0259; $\mathrm{R}_{f}=0.42 \quad(3: 7$ AcOEt/hexane). Endo-cycloadduct: Colorless needles; $\mathrm{mp}=83-86{ }^{\circ} \mathrm{C} ;[\alpha]_{\mathrm{D}}{ }^{26}=-58^{\circ}(c 1.01$, $\mathrm{CHCl}_{3}$ ); IR (KBr) 2955, 2895, 1709, 1599, 1489, 1458, 1402, 1379, 1314, 1290, 1248, 1217, 1155, $1134,1090,1073,1049,1024,1012,974,945,891,839,806 \mathrm{~cm}^{-1} ;{ }^{1} \mathrm{H}$ NMR $\left(400 \mathrm{MHz}, \mathrm{CDCl}_{3}\right) \delta=$ 


\section{Supporting Information}

$3.31(1 \mathrm{H}, \mathrm{dd}, J=6.3,10.7 \mathrm{~Hz}), 3.46(1 \mathrm{H}, \mathrm{dd}, J=4.4,10.7 \mathrm{~Hz}), 3.68(3 \mathrm{H}, \mathrm{s}), 4.26(1 \mathrm{H}, \mathrm{d}, J=12.0 \mathrm{~Hz})$, $4.35(1 \mathrm{H}, \mathrm{d}, J=12.0 \mathrm{~Hz}), 4.60-4.64(1 \mathrm{H}, \mathrm{m}), 4.96(1 \mathrm{H}, \mathrm{d}, J=5.6 \mathrm{~Hz}), 7.04(2 \mathrm{H}, \mathrm{d}, J=8.3 \mathrm{~Hz}), 7.39$ $(2 \mathrm{H}, \mathrm{d}, J=8.3 \mathrm{~Hz}), 7.41-7.45(1 \mathrm{H}, \mathrm{m}), 7.58-7.66(2 \mathrm{H}, \mathrm{m}), 7.90(1 \mathrm{H}, \mathrm{d}, J=7.8 \mathrm{~Hz})$; HRMS (EI) Found: 404.0280. Calcd for $\mathrm{C}_{19} \mathrm{H}_{17} \mathrm{BrO}_{5}\left(\mathrm{M}^{+}\right)$: 404.0259; $\mathrm{R}_{f}=0.38$ (3:7 AcOEt/hexane). The enantiomeric excess was determined by HPLC analysis (Daicel Chiralpak AS, 1:19 $i$-PrOH/hexane, Flow rate $=0.5 \mathrm{~mL} / \mathrm{min}, 35{ }^{\circ} \mathrm{C}$ ). Exo-isomer: $\mathrm{t}_{\mathrm{R}}=17.3 \mathrm{~min}$ (minor) and $21.4 \mathrm{~min}$ (major). Endoisomer: $\mathrm{t}_{\mathrm{R}}=14.6 \mathrm{~min}$ (minor) and $17.2 \mathrm{~min}$ (major).

5-Methyoxy-7-exo-methoxycarbonyl-7-endo-methyl-6,8-dioxabenzo[c]bicyclo[3.2.1]octan2-one and 5-Methyoxy-7-endo-methoxycarbonyl-7-exo-methyl-6,8-dioxabenzo[c]bicyclo[3.2.1]octan-2-one. A solution of $(S, S)$-Pybox- $i$ - $\operatorname{Pr}(15.1 \mathrm{mg}, 0.05 \mathrm{mmol})$ in $\mathrm{CH}_{2} \mathrm{Cl}_{2}(1.5 \mathrm{~mL})$ was added to a<smiles>COC1(OC)OC(C)(C(C)=O)C(I)C(=O)c2ccccc21</smiles>
suspension of $\mathrm{Sc}(\mathrm{OTf})_{3}(24.6 \mathrm{mg}, 0.05 \mathrm{mmol})$ and $\mathrm{MS} 4 \mathrm{~A}(0.50 \mathrm{~g})$ in $\mathrm{CH}_{2} \mathrm{Cl}_{2}$ $(1.0 \mathrm{~mL})$. After stirring the mixture for $2 \mathrm{~h}, \mathrm{Rh}_{2}(\mathrm{OAc})_{4}(4.4 \mathrm{mg}, 0.01 \mathrm{mmol})$, a solution of methyl pyruvate (9) $(102.1 \mathrm{mg}, 90 \mu \mathrm{L}, 1.0 \mathrm{mmol})$ in $\mathrm{CH}_{2} \mathrm{Cl}_{2}(2.5$ $\mathrm{mL}$ ), and pyruvic acid ( $1 \mathrm{M}$ solution in $\left.\mathrm{CH}_{2} \mathrm{Cl}_{2}, 50 \mu \mathrm{L}, 0.05 \mathrm{mmol}\right)$ were successively added. The mixture was cooled to $-10{ }^{\circ} \mathrm{C}$, and then a solution of diazoacetophenone 1 (102.1 $\mathrm{mg}, 0.5 \mathrm{mmol})$ in $\mathrm{CH}_{2} \mathrm{Cl}_{2}(5 \mathrm{~mL})$ was added over a period of $1 \mathrm{~h}$. After removal of MS 4A through celite, the reaction mixture was filtered through a plug of silica gel $(3 \mathrm{~cm})$ with AcOEt/hexane $(1: 1,80 \mathrm{~mL})$ as an eluent. The solvent was removed in vacuo, and the residue was purified by MPLC (3:97 - 5:95 AcOEt/hexane) to provide $118.2 \mathrm{mg}(84 \%)$ of exocycloadduct and $5.3 \mathrm{mg}$ (4\%) of endo-cycloadduct. Exo-cycloadduct: Colorless prisms; $\mathrm{mp}=84-86$ ${ }^{\circ} \mathrm{C} ;[\alpha]_{\mathrm{D}}{ }^{23}=-133^{\circ}\left(c 1.01, \mathrm{CHCl}_{3}\right)$; IR (KBr) 3015, 2996, 2963, 1755, 1703, 1601, 1458, 1443, 1377 , $1319,1300,1283,1231,1184,1146,1119,1094,1076,1047,1026,976,945,909 \mathrm{~cm}^{-1} ;{ }^{1} \mathrm{H}$ NMR $(400$ $\left.\mathrm{MHz}, \mathrm{CDCl}_{3}\right) \delta=1.31(3 \mathrm{H}, \mathrm{s}), 3.74(3 \mathrm{H}, \mathrm{s}), 3.87(3 \mathrm{H}, \mathrm{s}), 5.22(1 \mathrm{H}, \mathrm{s}), 7.48-7.53(1 \mathrm{H}, \mathrm{m}), 7.62-7.68$ $(2 \mathrm{H}, \mathrm{m}), 8.00(1 \mathrm{H}, \mathrm{d}, J=7.6 \mathrm{~Hz})$. Found: $\mathrm{C}, 60.40 ; \mathrm{H}, 5.04 \%$. Calcd for $\mathrm{C}_{14} \mathrm{H}_{14} \mathrm{O}_{6}: \mathrm{C}, 60.43 ; \mathrm{H}, 5.07 \%$. $\mathrm{R}_{f}=0.55$ (3:7 AcOEt/hexane). Endo-cycloadduct: Colorless prisms; $\mathrm{mp}=60-62{ }^{\circ} \mathrm{C}$; IR (KBr) 3007, $2961,1752,1705,1603,1464,1453,1312,1279,1254,1194,1132,1090,1073,1055,1042 \mathrm{~cm}^{-1} ;{ }^{1} \mathrm{H}$ $\operatorname{NMR}\left(400 \mathrm{MHz}, \mathrm{CDCl}_{3}\right) \delta=1.84(3 \mathrm{H}, \mathrm{s}), 3.55(3 \mathrm{H}, \mathrm{s}), 3.73(3 \mathrm{H}, \mathrm{s}), 4.74(1 \mathrm{H}, \mathrm{s}), 7.46-7.50(1 \mathrm{H}, \mathrm{m})$, $7.61-7.68(2 \mathrm{H}, \mathrm{m}), 7.92(1 \mathrm{H}, \mathrm{d}, J=7.8 \mathrm{~Hz})$; Found: C, 60.58; H, 5.04\%. Calcd for $\mathrm{C}_{14} \mathrm{H}_{14} \mathrm{O}_{6}: \mathrm{C}$, $60.43 ; \mathrm{H}, 5.07 \% . \quad \mathrm{R}_{f}=0.45$ (3:7 AcOEt/hexane). The enantiomeric excess was determined by HPLC analysis (Daicel Chiralpak AS, 1:19 $i-\mathrm{PrOH} /$ hexane, Flow rate $=0.5 \mathrm{~mL} / \mathrm{min}, 35^{\circ} \mathrm{C}$ ). Exo-isomer: $\mathrm{t}_{\mathrm{R}}=$ 10.7 min (minor) and 12.4 min (major). Endo-isomer: $t_{R}=13.1$ min (minor) and 17.2 min (major).

\section{7-Exo-(benzyloxy)carbonyl-5-methyoxy-7-endo-methyl-6,8-dioxabenzo[c]bicyclo[3.2.1]-} octan-2-one and 7-Endo-(benzyloxy)carbonyl-5-methyoxy-7-exo-methyl-6,8-dioxabenzo[c]bicyclo[3.2.1]octan-2-one. A solution of $(S, S)$-Pybox- $i-\operatorname{Pr}(15.1 \mathrm{mg}, 0.05 \mathrm{mmol})$ in $\mathrm{CH}_{2} \mathrm{Cl}_{2}(1.5 \mathrm{~mL})$ was<smiles>COC1(OC)OC(C)(C(=O)OCc2ccccc2)C(I)C(=O)c2ccccc21</smiles>
added to a suspension of $\mathrm{Sc}(\mathrm{OTf})_{3}(24.6 \mathrm{mg}, 0.05 \mathrm{mmol})$ and $\mathrm{MS} 4 \mathrm{~A}(0.50 \mathrm{~g})$ in $\mathrm{CH}_{2} \mathrm{Cl}_{2}(1.0 \mathrm{~mL})$. After stirring the mixture for $2 \mathrm{~h}, \mathrm{Rh}_{2}(\mathrm{OAc})_{4}(4.4 \mathrm{mg}, 0.01$ mmol), a solution of benzyl pyruvate (10) $(178.2 \mathrm{mg}, 1.0 \mathrm{mmol})$ in $\mathrm{CH}_{2} \mathrm{Cl}_{2}(2.5$ $\mathrm{mL}$ ), and pyruvic acid ( $1 \mathrm{M}$ solution in $\mathrm{CH}_{2} \mathrm{Cl}_{2}, 0.1 \mathrm{~mL}, 0.1 \mathrm{mmol}$ ) were successively added. The mixture was cooled to $-10{ }^{\circ} \mathrm{C}$, and then a solution of diazoacetophenone 1 (102.1 $\mathrm{mg}, 0.5 \mathrm{mmol})$ in $\mathrm{CH}_{2} \mathrm{Cl}_{2}(5 \mathrm{~mL})$ was added over a period of $1 \mathrm{~h}$. After removal of MS 4A through celite, the reaction mixture was filtered through a plug of silica gel $(3 \mathrm{~cm})$ with AcOEt/hexane $(1: 1,80 \mathrm{~mL})$ as an eluent. The solvent was removed in vacuo, and the residue was purified by MPLC (3:97 - 5:95 AcOEt/hexane) to provide $10.8 \mathrm{mg}(6 \%)$ of endo- 


\section{Supporting Information}

cycloadduct and $204.6 \mathrm{mg}$ of a mixture (54:46) of exo-cycloadduct and benzyl pyruvate. The yield of exo-cycloadduct was calculated from ${ }^{1} \mathrm{H}$ NMR (143.9 mg, 82\%). Exo-cycloadduct: Colorless oil; $[\alpha]_{\mathrm{D}}^{28}=-103^{\circ}\left(c 1.14, \mathrm{CHCl}_{3}\right.$, containing benzyl pyruvate $\left.(21 \mathrm{wt} \%)\right)$; IR (Neat) 3034, 2959, 1732, $1711,1603,1458,1381,1360,1292,1262,1219,1136,1092,1078,1047,1028 \mathrm{~cm}^{-1}$; ${ }^{1} \mathrm{H}$ NMR (400 $\left.\mathrm{MHz}, \mathrm{CDCl}_{3}\right) \delta=1.30(3 \mathrm{H}, \mathrm{s}), 3.62(3 \mathrm{H}, \mathrm{s}), 5.23(1 \mathrm{H}, \mathrm{s}), 5.24(1 \mathrm{H}, \mathrm{d}, J=12.1 \mathrm{~Hz}), 5.33(1 \mathrm{H}, \mathrm{d}, J=$ $12.1 \mathrm{~Hz}), 7.36-7.40(5 \mathrm{H}, \mathrm{m}), 7.47-7.51(1 \mathrm{H}, \mathrm{m}), 7.60-7.66(2 \mathrm{H}, \mathrm{m}), 7.99(1 \mathrm{H}, \mathrm{d}, J=7.6 \mathrm{~Hz}) ;{ }^{13} \mathrm{C}$ NMR $\left(100 \mathrm{MHz}, \mathrm{CDCl}_{3}\right) \delta=19.8,50.4,67.9,79.2,85.4,118.9,122.7,126.4,128.2,128.5,128.7$, 128.8, 129.5, 134.8, 135.0, 143.2, 171.3, 191.5; HRMS (EI) Found: 354.1072. Calcd for $\mathrm{C}_{20} \mathrm{H}_{18} \mathrm{O}_{6}$ $\left(\mathrm{M}^{+}\right): 354.1102 ; \mathrm{R}_{f}=0.53$ (3:7 AcOEt/hexane). Endo-cycloadduct: Colorless oil; IR (Neat) 3069, 3034, 2944, 2955, 1736, 1709, 1605, 1499, 1458, 1377, 1292, 1248, 1215, 1132, 1088, 1074, 1045, 951, 887 $\mathrm{cm}^{-1} ;{ }^{1} \mathrm{H}$ NMR $\left(400 \mathrm{MHz}, \mathrm{CDCl}_{3}\right) \delta=1.84(3 \mathrm{H}, \mathrm{s}), 3.72(3 \mathrm{H}, \mathrm{s}), 4.74(1 \mathrm{H}, \mathrm{s}), 4.87(1 \mathrm{H}, \mathrm{d}, J=12.2 \mathrm{~Hz})$, $5.01(1 \mathrm{H}, \mathrm{d}, J=12.2 \mathrm{~Hz}), 7.06-7.08(2 \mathrm{H}, \mathrm{m}), 7.25-7.29(3 \mathrm{H}, \mathrm{m}), 7.36-7.40(1 \mathrm{H}, \mathrm{m}), 7.57-7.66$ $(2 \mathrm{H}, \mathrm{m}), 7.76(1 \mathrm{H}, \mathrm{d}, J=7.8 \mathrm{~Hz})$; HRMS (EI) Found: 354.1097. Calcd for $\mathrm{C}_{20} \mathrm{H}_{18} \mathrm{O}_{6}\left(\mathrm{M}^{+}\right): 354.1102 ; \mathrm{R}_{f}$ $=0.44(3: 7 \mathrm{AcOEt} /$ hexane). The enantiomeric excess was determined by HPLC analysis. Exo-isomer (Daicel Chiralpak AD, 1:19 $i$-PrOH/hexane, Flow rate $=0.5 \mathrm{~mL} / \mathrm{min}, 35{ }^{\circ} \mathrm{C}$ ): $\mathrm{t}_{\mathrm{R}}=17.0 \mathrm{~min}$ (minor) and $21.6 \mathrm{~min}$ (major). Endo-isomer (Daicel Chiralpak AS, 1:19 $i$-PrOH $/$ hexane, Flow rate $=0.5 \mathrm{~mL} / \mathrm{min}$, $35^{\circ} \mathrm{C}$ ): $\mathrm{t}_{\mathrm{R}}=13.3 \mathrm{~min}$ (minor) and $17.6 \mathrm{~min}$ (major).

\section{5,8-Epoxy-5-methoxy-6-exo-(2-oxazolidinoyl)carbonyl-9-oxo-6,7,8,9-tetrahydro-5H-} benzocycloheptene and 5,8-Epoxy-5-methoxy-6-endo-(2-oxazolidinoyl)carbonyl-9-oxo-6,7,8,9tetrahydro-5 $\boldsymbol{H}$-benzocycloheptene. A solution of 2,6-bis[(4S)-(-)-4-phenyl-2-oxazolin-2-yl]pyridine

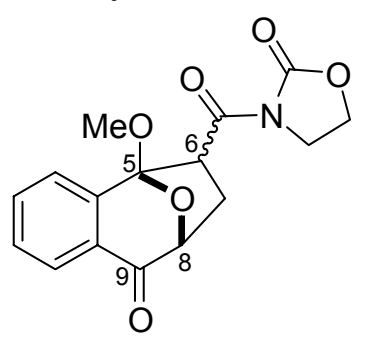
$((S, S)$-Pybox-Ph, $18.5 \mathrm{mg}, 0.05 \mathrm{mmol})$ in $\mathrm{CH}_{2} \mathrm{Cl}_{2}(1.5 \mathrm{~mL})$ was added to a suspension of $\mathrm{Yb}(\mathrm{OTf})_{3}(31.0 \mathrm{mg}, 0.05 \mathrm{mmol})$ and $\mathrm{MS} 4 \mathrm{~A}(0.50 \mathrm{~g})$ in $\mathrm{CH}_{2} \mathrm{Cl}_{2}$ $(1.0 \mathrm{~mL})$. After stirring the mixture for $2 \mathrm{~h}, \mathrm{Rh}_{2}(\mathrm{OAc})_{4}(4.4 \mathrm{mg}, 0.01 \mathrm{mmol})$ and a solution of 3-acryloyl-2-oxazolidinone (11) (144.1 $\mathrm{mg}, 1.0 \mathrm{mmol}$ ) in $\mathrm{CH}_{2} \mathrm{Cl}_{2}$ $(2.5 \mathrm{~mL})$ were successively added. The mixture was cooled to $-25{ }^{\circ} \mathrm{C}$, and then a solution of diazoacetophenone $1(102.1 \mathrm{mg}, 0.5 \mathrm{mmol})$ in $\mathrm{CH}_{2} \mathrm{Cl}_{2}(5 \mathrm{~mL})$ was added over a period of $6 \mathrm{~h}$. After removal of MS 4A through celite, the reaction mixture was filtered through a plug of silica gel $(3 \mathrm{~cm})$ with AcOEt/hexane $(1: 1$, $80 \mathrm{~mL})$ as an eluent. The solvent was removed in vacuo, and the residue was purified by column chromatography (3:7 - 1:1 AcOEt/hexane) to provide $141.6 \mathrm{mg}(89 \%)$ of a mixture of exo- and endocycloadducts. The exo/endo ratio was determined by ${ }^{1} \mathrm{H}$ NMR analysis (exo:endo $=88: 12$ ). Colorless solid; $[\alpha]_{\mathrm{D}}{ }^{25}=+52^{\circ}\left(c 0.99, \mathrm{CHCl}_{3}\right.$, A mixture of exo- and endo-cycloadducts (88:12)); IR (KBr, A mixture of exo- and endo-cycloadducts) 2976, 2955, 2926, 2874, 1780, 1688, 1601, 1472, 1456, 1440, 1385, 1316, 1302, 1287, 1263, 1223, 1161, 1121, 1096, 1073, 1049, 1022, 1009, $978 \mathrm{~cm}^{-1}$; Found: C, 60.59; H, 4.72; N, 4.24\%. Calcd for $\mathrm{C}_{16} \mathrm{H}_{15} \mathrm{NO}_{6}$ : C, 60.57; H, 4.77; N, 4.41\%. Exo-cycloadduct: ${ }^{1} \mathrm{H}$ $\operatorname{NMR}\left(\mathrm{CDCl}_{3}, 400 \mathrm{MHz}\right) \delta=1.82(1 \mathrm{H}, \mathrm{ddd}, J=1.7,9.0,13.7 \mathrm{~Hz}), 3.13(1 \mathrm{H}, \mathrm{ddd}, J=3.9,9.3,13.7 \mathrm{~Hz})$, $3.33(3 \mathrm{H}, \mathrm{s}), 3.9-4.0(1 \mathrm{H}, \mathrm{m}), 4.1-4.2(1 \mathrm{H}, \mathrm{m}), 4.2-4.5(2 \mathrm{H}, \mathrm{m}), 4.58(1 \mathrm{H}, \mathrm{dd}, J=3.9,9.0 \mathrm{~Hz}), 4.88$ $(1 \mathrm{H}, \mathrm{dd}, J=1.7,9.3 \mathrm{~Hz}), 7.3-8.0(4 \mathrm{H}, \mathrm{m}, \mathrm{ArH}) ;{ }^{13} \mathrm{C} \mathrm{NMR}\left(100 \mathrm{MHz}, \mathrm{CDCl}_{3}\right) \delta=28.6,43.0,49.0$, 53.9, 62.0, 80.2, 108.5, 124.7, 127.2, 129.2, 129.7, 134.3, 143.0, 153.1, 170.5, 194.6; $\mathrm{R}_{f}=0.51$ (ether). Endo-cycloadduct: ${ }^{1} \mathrm{H}$ NMR $\left(\mathrm{CDCl}_{3}, 400 \mathrm{MHz}\right) \delta=2.22(1 \mathrm{H}$, ddd, $J=1.5,5.6,13.4 \mathrm{~Hz}), 2.72(1 \mathrm{H}$, ddd, $J=8.9,10.9,13.4 \mathrm{~Hz}), 3.4-3.5(1 \mathrm{H}, \mathrm{m}), 3.52(3 \mathrm{H}, \mathrm{s}), 3.6-3.8(1 \mathrm{H}, \mathrm{m}), 4.2-4.5(2 \mathrm{H}, \mathrm{m}), 4.78$ $(1 \mathrm{H}, \mathrm{dd}, J=1.5,8.9 \mathrm{~Hz}), 5.00(1 \mathrm{H}, \mathrm{dd}, J=5.6,10.9 \mathrm{~Hz}), 7.3-8.0(4 \mathrm{H}, \mathrm{m}) ;{ }^{13} \mathrm{C} \mathrm{NMR}(100 \mathrm{MHz}$, $\left.\mathrm{CDCl}_{3}\right) \delta=29.6,43.2,46.8,51.8,61.9,79.9,108.2,123.4,126.9,129.2,130.2,133.4,142.3,153.1$, 170.5, 194.3; $\mathrm{R}_{f}=0.45$ (ether). The enantiomeric excess was determined by HPLC analysis (Daicel 


\section{Supporting Information}

Chiralpak AS, 1:4 $i$-PrOH/hexane, Flow rate $=0.5 \mathrm{~mL} / \mathrm{min}, 35^{\circ} \mathrm{C}$ ). Exo-isomer: $\mathrm{t}_{\mathrm{R}}=25.9$ min (major) and $30.1 \mathrm{~min}$ (minor). Endo-isomer: $\mathrm{t}_{\mathrm{R}}=55.1 \mathrm{~min}$ (major) and $120.9 \mathrm{~min}$ (minor).

\section{References and Notes}

1) (a) Suga, H.; Kakehi, A.; Ito, S.; Inoue, K.; Ishida, H.; Ibata, T. Org. Lett., 2000, 2, 3145. (b) Suga, H.; Kakehi, A.; Ito, S.; Inoue, K.; Ishida, H.; Ibata, T. Bull. Chem. Soc. Jpn., 2001, 74, 1115. (c) Ueda, K.; Ibata, T.; Takebayashi, M. Bull. Chem. Soc. Jpn. 1972, 45, 2779.

2) (a) Arndt, H. C.; Carroll, S. A. Synthesis, 1979, 202. (b) Evans, D. A.; Kozlowski, M. C.; Murry, J. A.; Burgey, C. S.; Campos, K. R.; Connell, B. T.; Staples, R. J. J. Am. Chem. Soc. 1999, 121, 669 and references therein.

3) Yamamoto, Y.; Maruyama, K.; Komatsu, T.; Ito, W. J. Org. Chem. 1986, 51, 886.

4) Evans, D. A.; Miller, S. J.; Lectka, T.; von Matt, P. J. Am. Chem. Soc. 1999, 121, 7559.

5) (S,S)-Pybox-i-Pr: (a) Totleben, M. J.; Prasad, J. S.; Simpson, J. H.; Chan, S. H.; Vanyo, D. J.; Kuehner, D. E.; Deshpande, R.; Kodersha, G. A. J. Org. Chem. 2001, 66, 1057. (b) Desimoni, G.; Faita, G.; Filippone, S.; Mella, M.; Zampori, M. G.; Zema, M. Tetrahedron, 2001, 57, 10203. (S,S)-Pybox-Ph: (c) Davies, I. W.; Gerena, L.; Lu, N.; Larsen, R. D.; Reider, P. J. J. Org. Chem. 1996, 61, 9629. (S,S)-Pybox-TBSm: (d) Iwasa, S.; Nakamura, H.; Nishiyama, H. Heterocycles, 2000, 52, 939. 


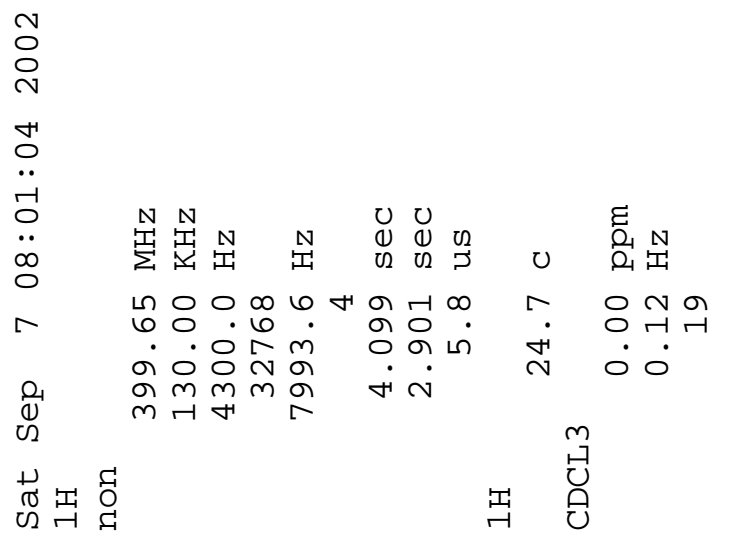

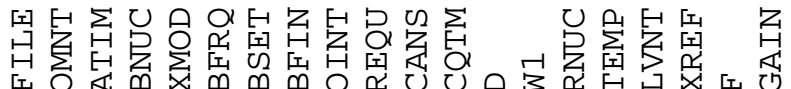

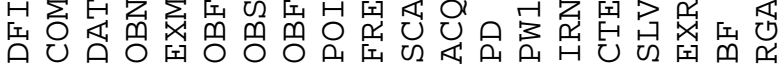

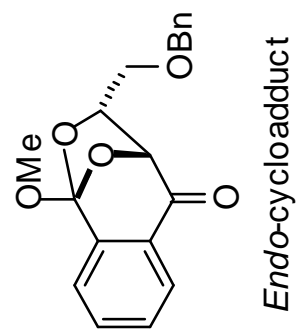

$000^{\circ} 0$

$082^{\cdot} \varepsilon$

$\angle 0 \varepsilon^{\circ} \varepsilon$

$\varepsilon 乙 \varepsilon^{\circ} \varepsilon$

$\angle \varepsilon \sigma^{\circ} \varepsilon-$

$8 \nabla \nabla^{\circ} \varepsilon$

$\nabla 9 \sigma^{\circ} \varepsilon$

$\bar{\nabla} \sigma^{\circ} \varepsilon-$

$\varepsilon 89^{\circ} \varepsilon$

$90 \varepsilon^{\circ} \nabla$

ธع $\varepsilon^{\circ} \hbar$

$80 \sigma \cdot \hbar$

8Eゥ.

$\left.809^{\circ} \cdot 7\right]=$

टरे $\overline{9}^{\circ}$

$\varepsilon \varepsilon 9^{\circ} \emptyset$

$879^{\circ}$

$9 S 6^{\circ} \circ$

$88 T^{\circ}$

$66 I \cdot L-L$

$\varepsilon 0 \tau^{\circ} L$

$\nabla \varepsilon \tau^{\circ} L$

$6 \varepsilon \tau^{\circ}\llcorner$

Zऽ $\cdot L$

$\angle S Z^{\circ} L-$

[9 $Z^{\circ} L-$

$08 Z^{\circ} \cdot L$

$962^{\circ} L \square$

乙oर

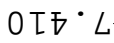

$82 \sigma^{\circ} L$

$9 \nabla \sigma^{\circ} L$

$\angle \nabla \sigma \cdot L$

$\nabla \angle S^{\circ} \angle$

D6 $9^{\circ}$

2 L $9^{\circ} \mathrm{L}$

Iฤ $9^{\circ} L$

$\varepsilon \mp 9^{\circ} L$

$099^{\circ} L$

0 I6.

$626^{\circ} \mathrm{L}$

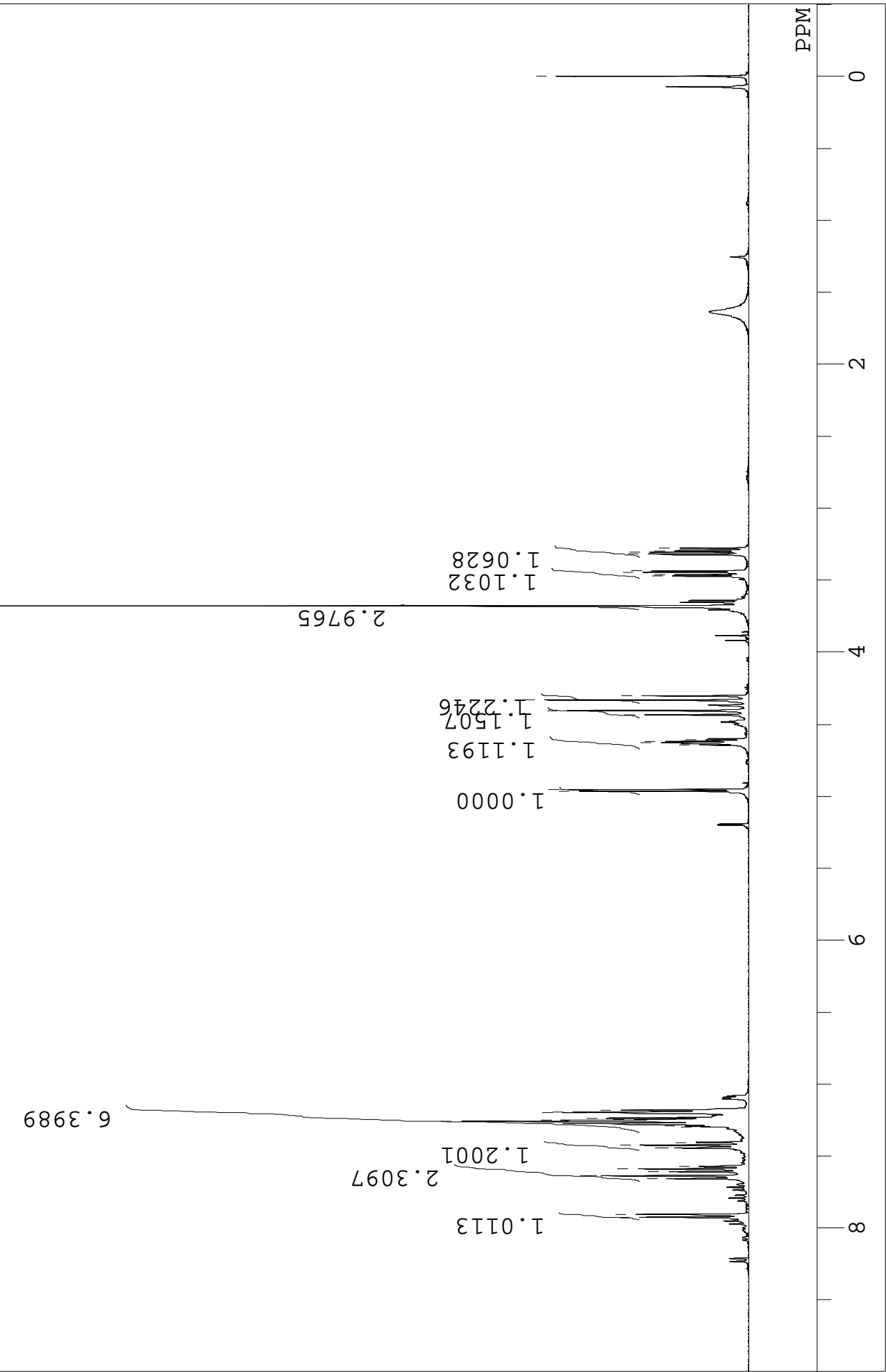




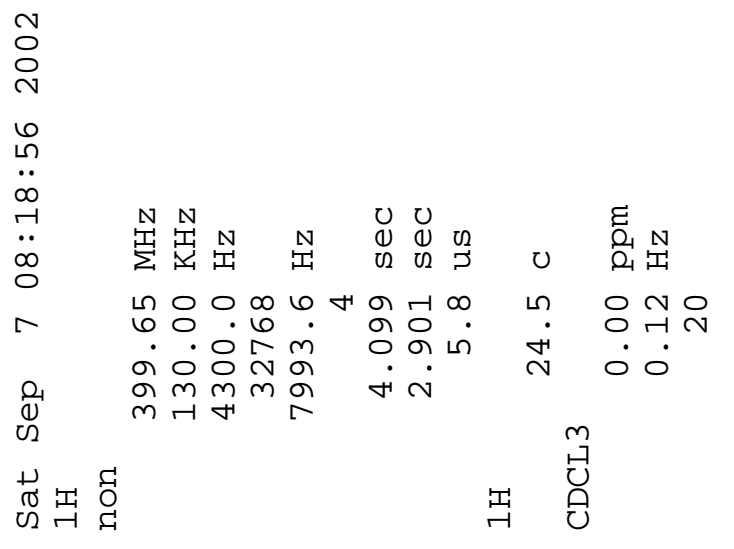

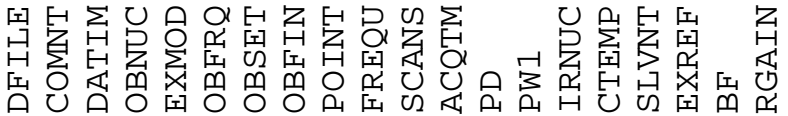
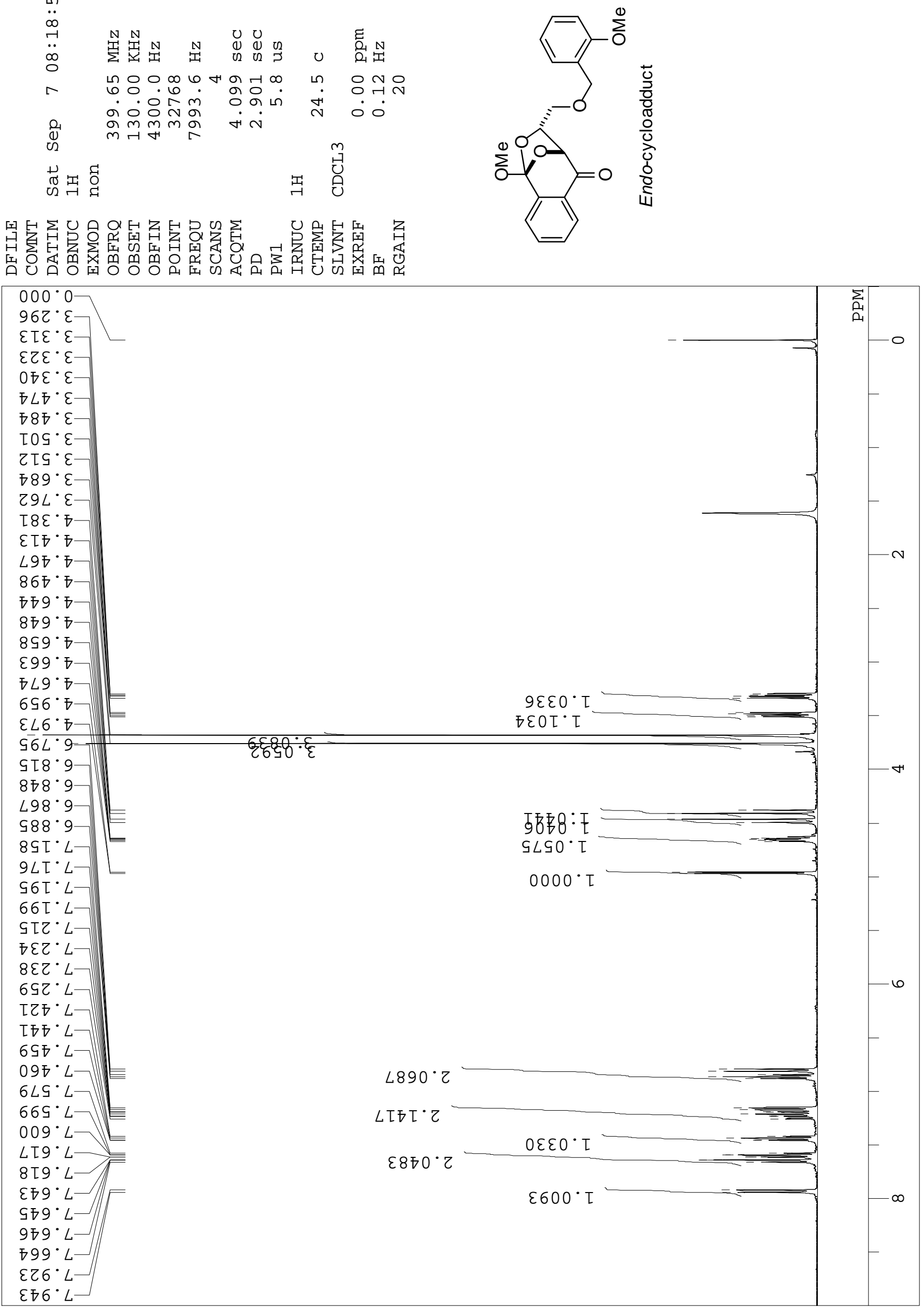


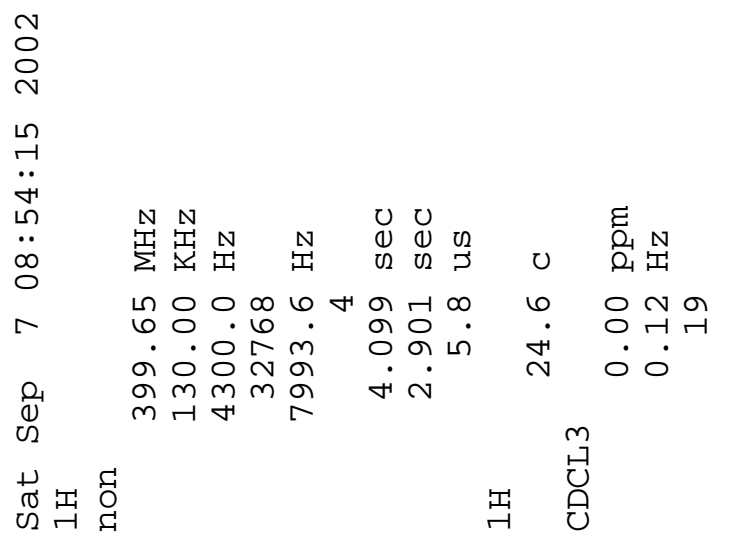

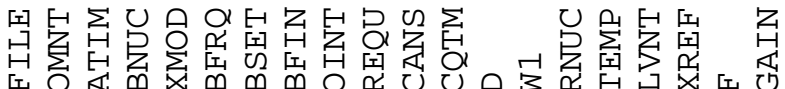

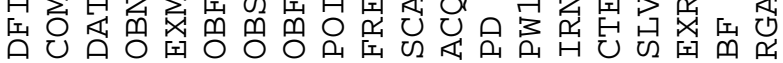
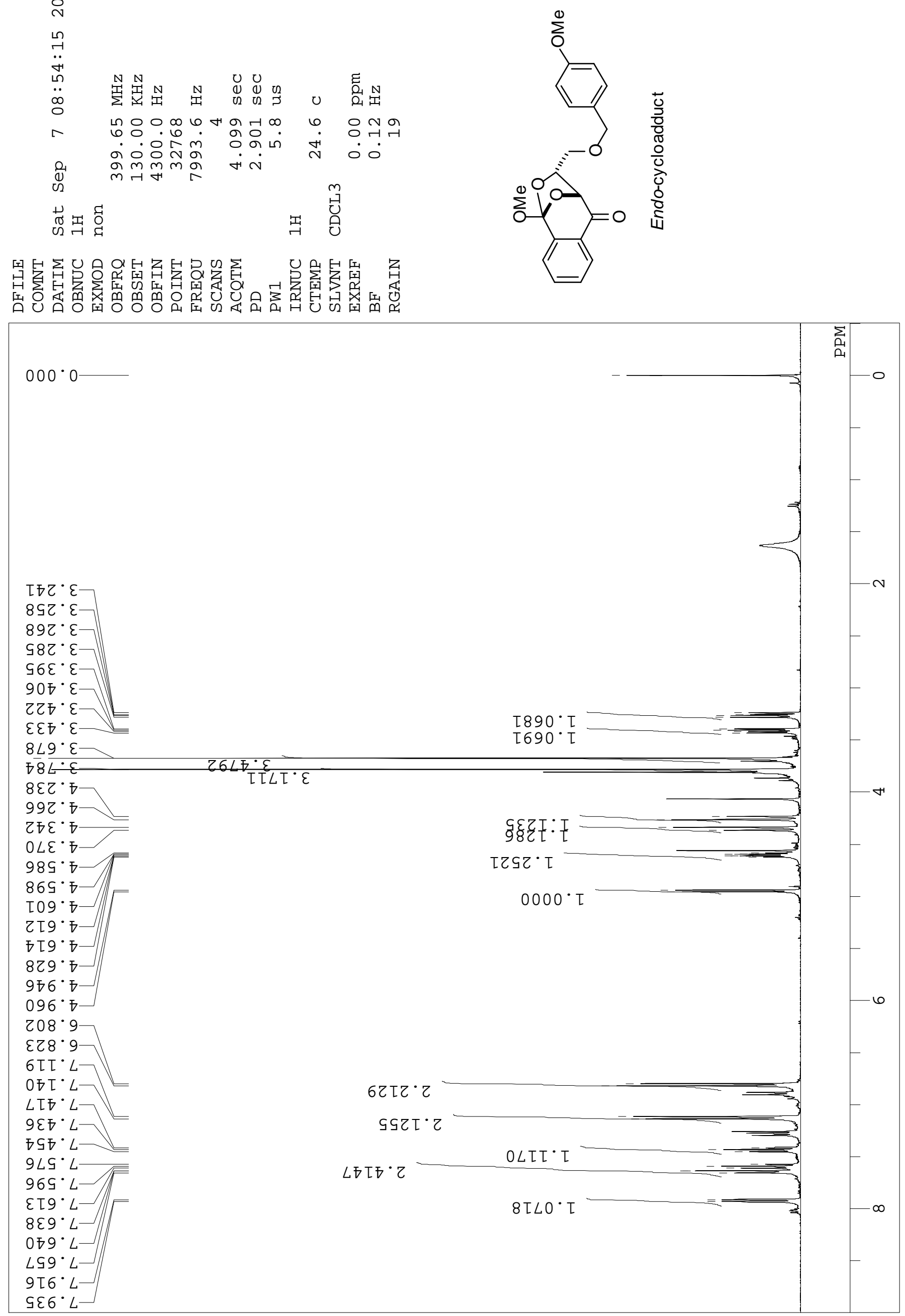


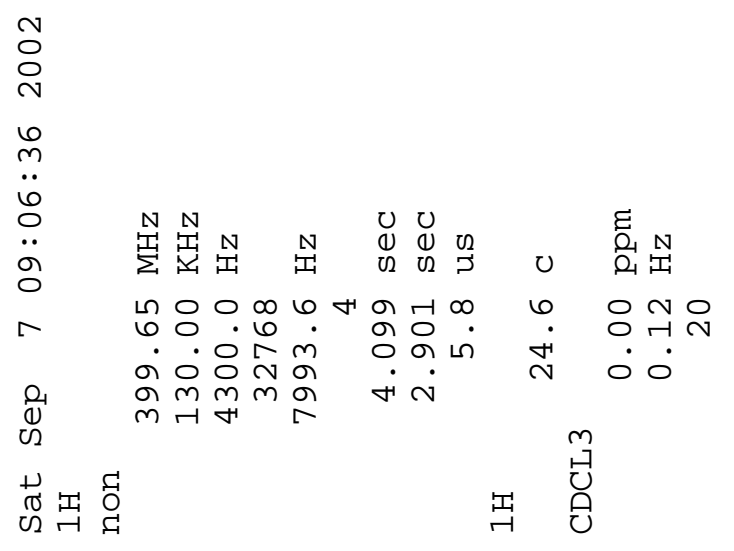

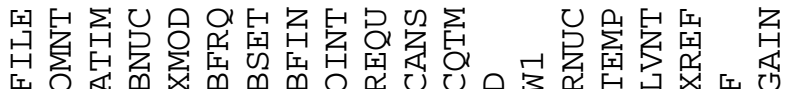

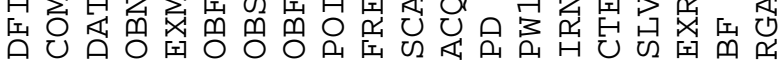
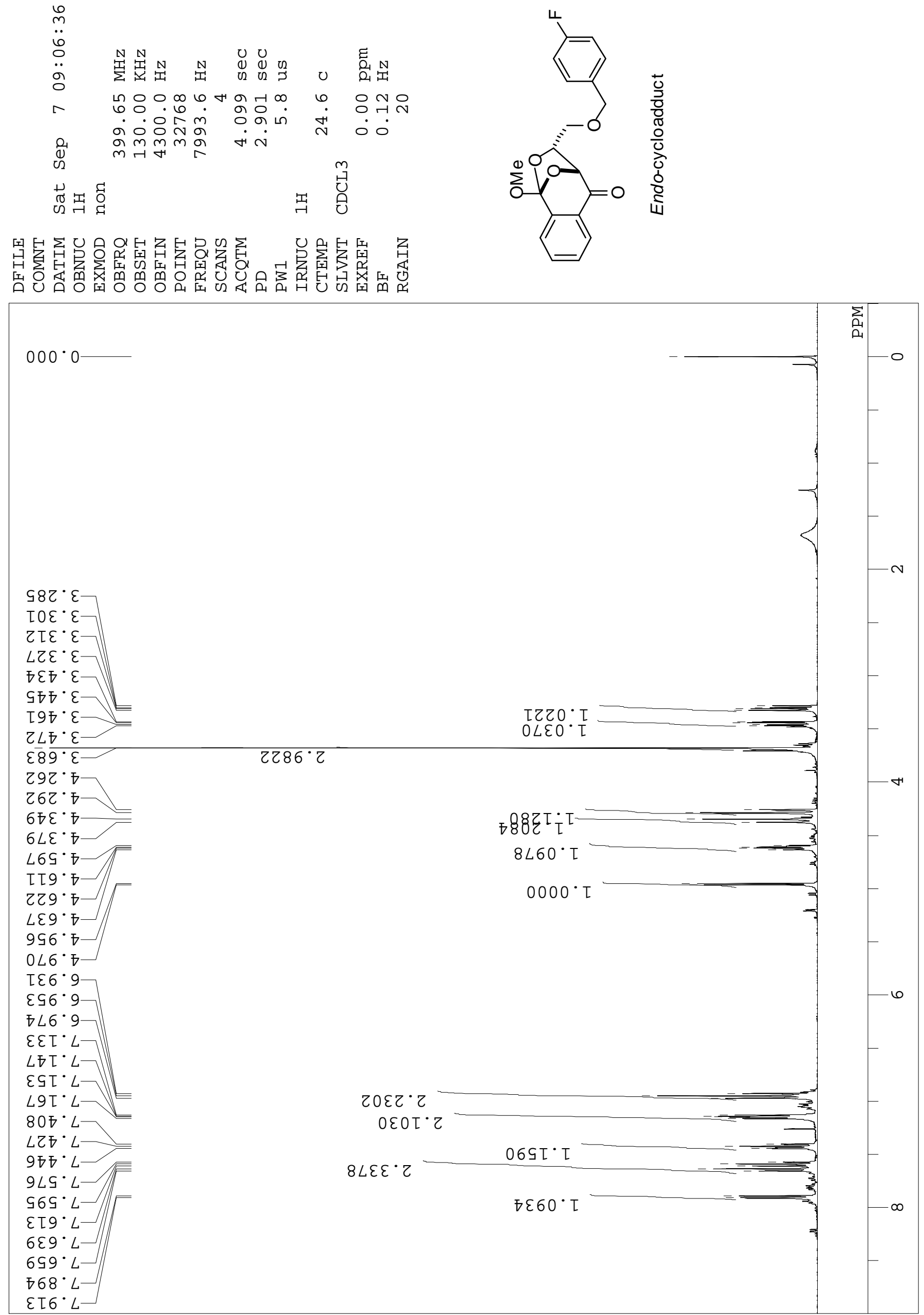


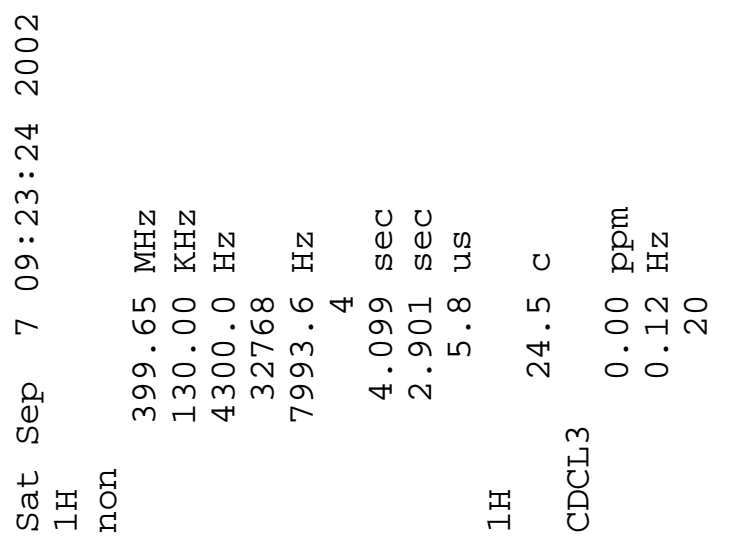

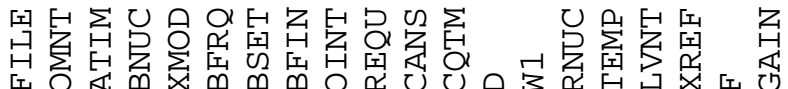

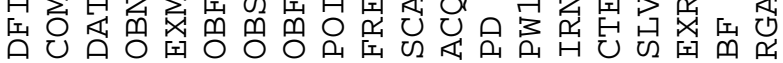
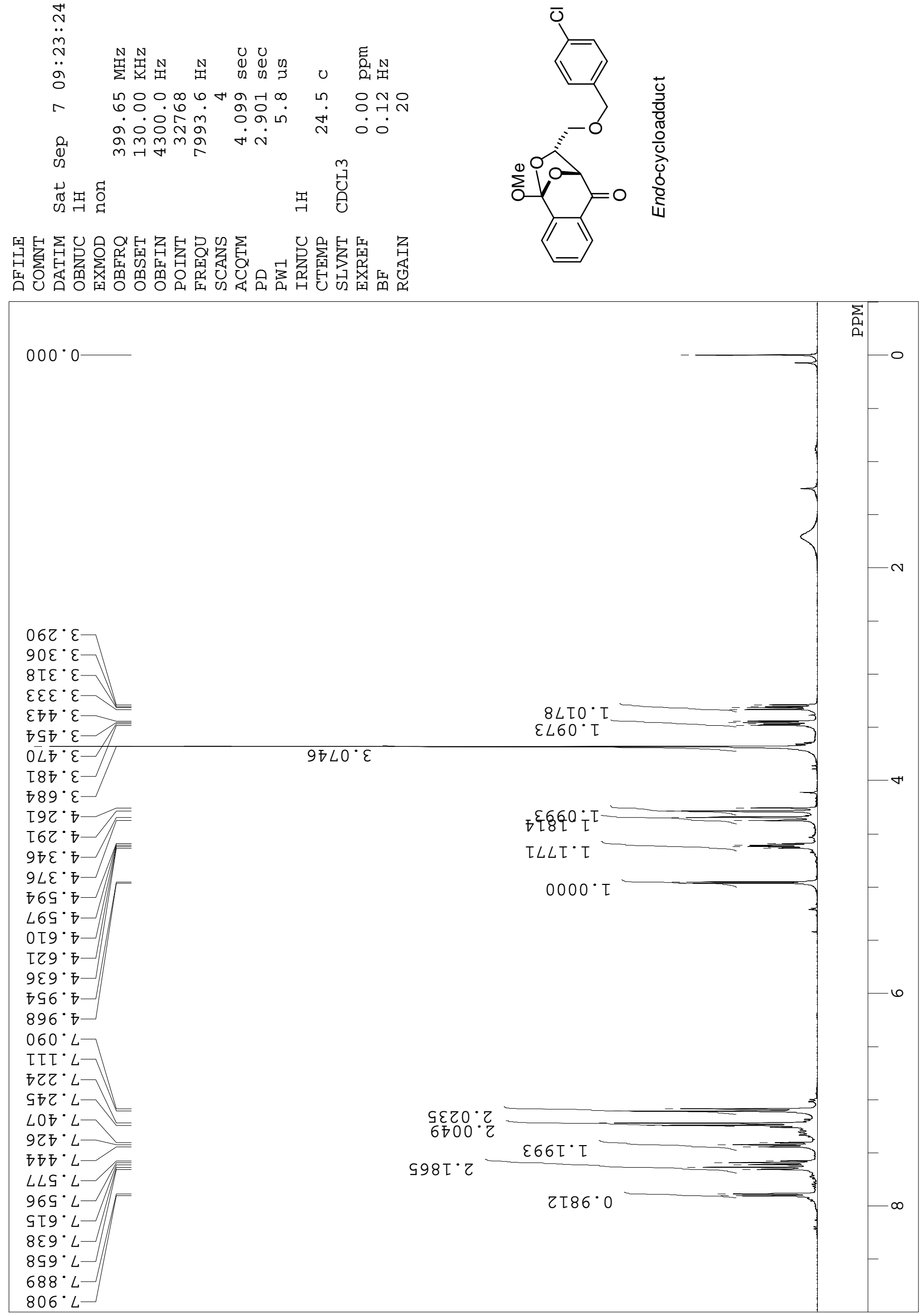


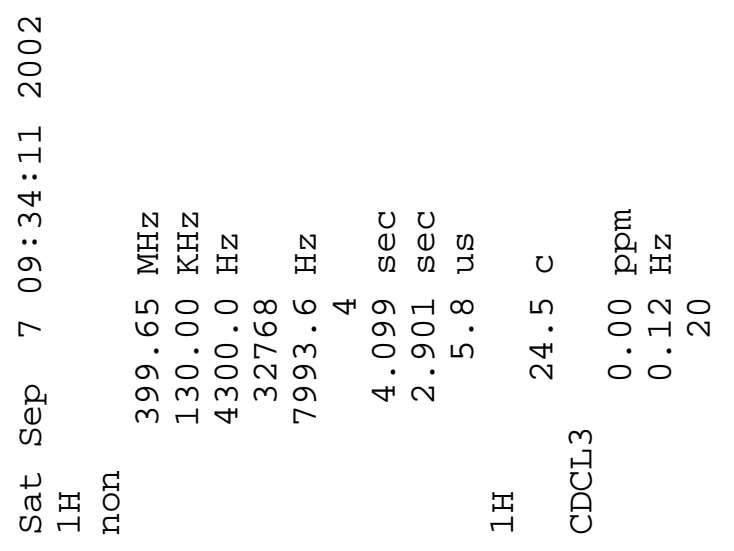

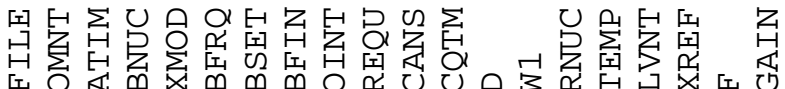

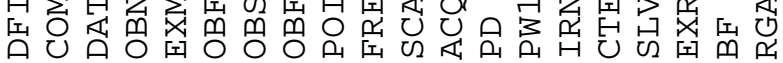
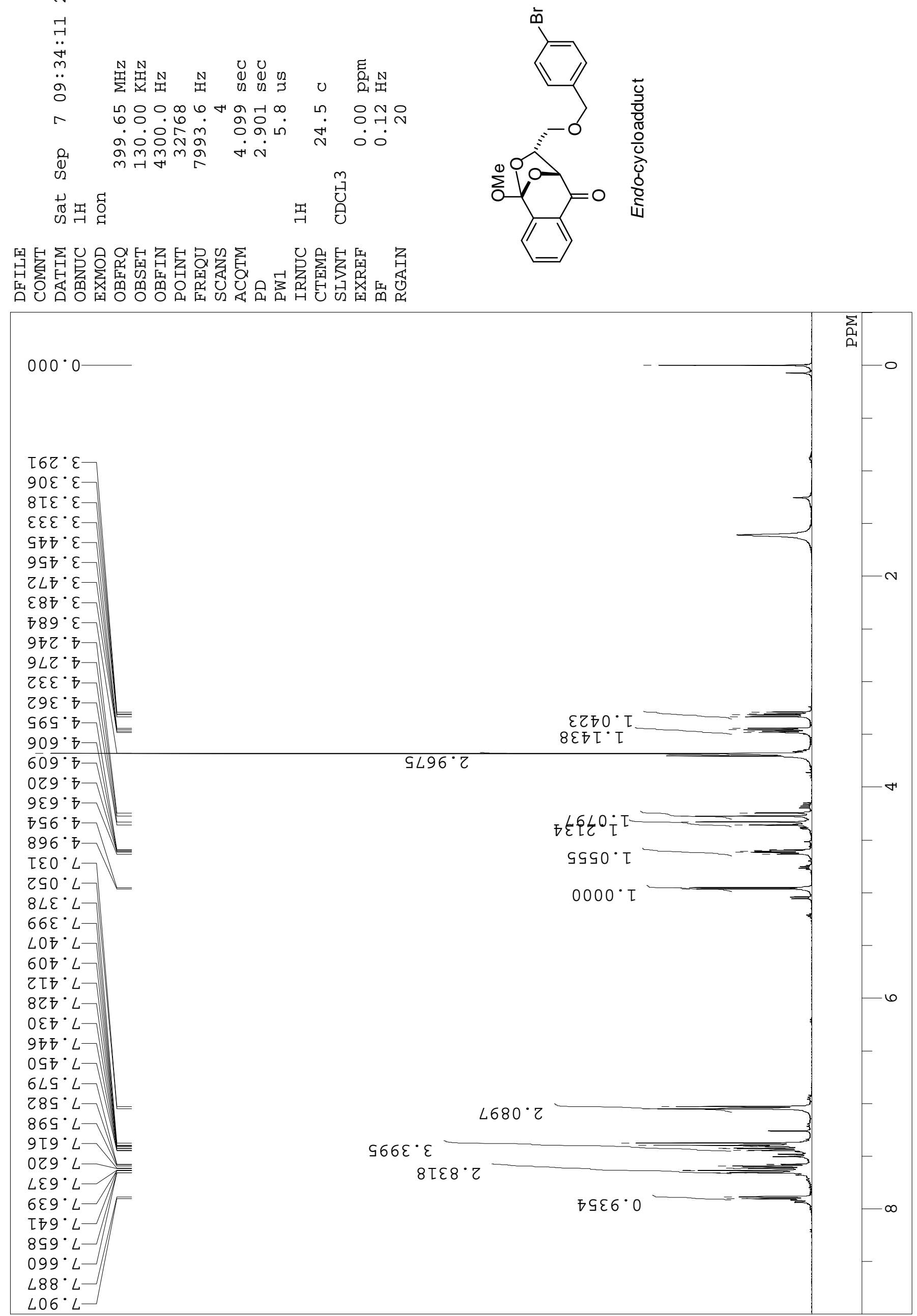
$\stackrel{\sim}{\circ}$

ำ

$\ddot{\sim}$

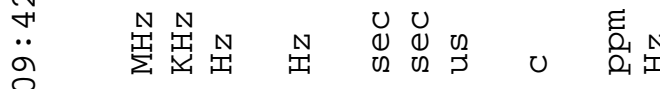

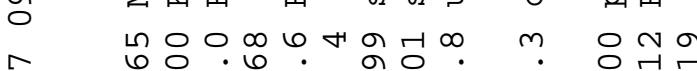

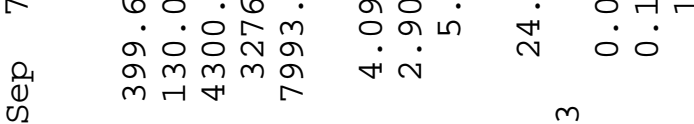

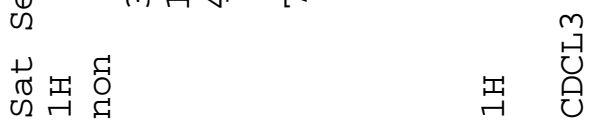

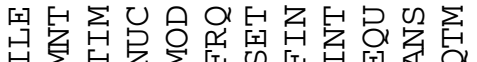

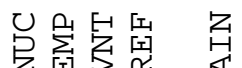

仓

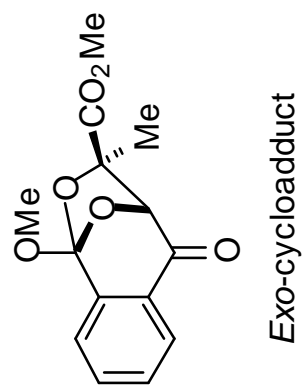

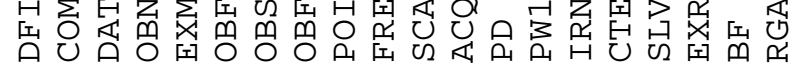

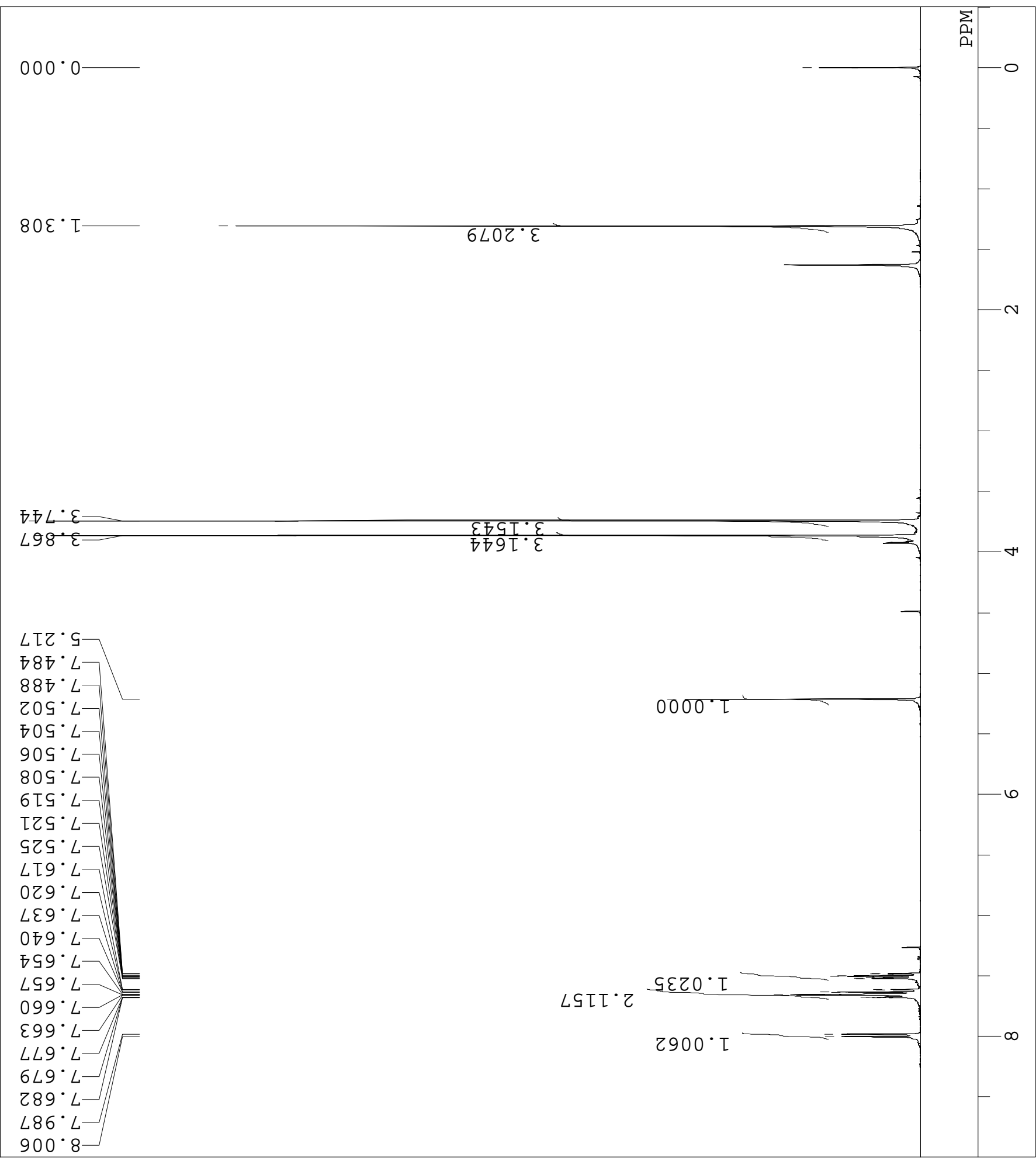



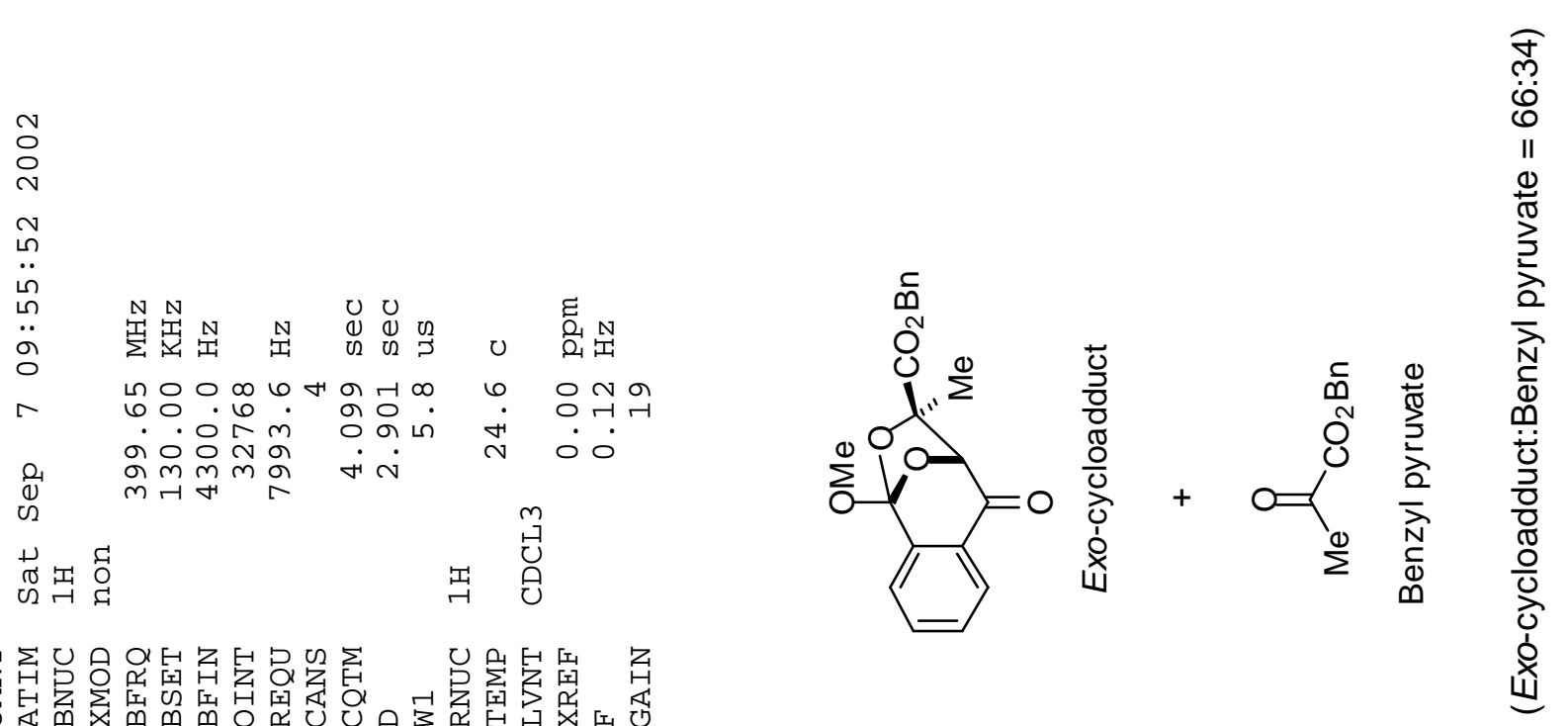

$000 \cdot 0$

๑० $\varepsilon^{\circ} \tau$

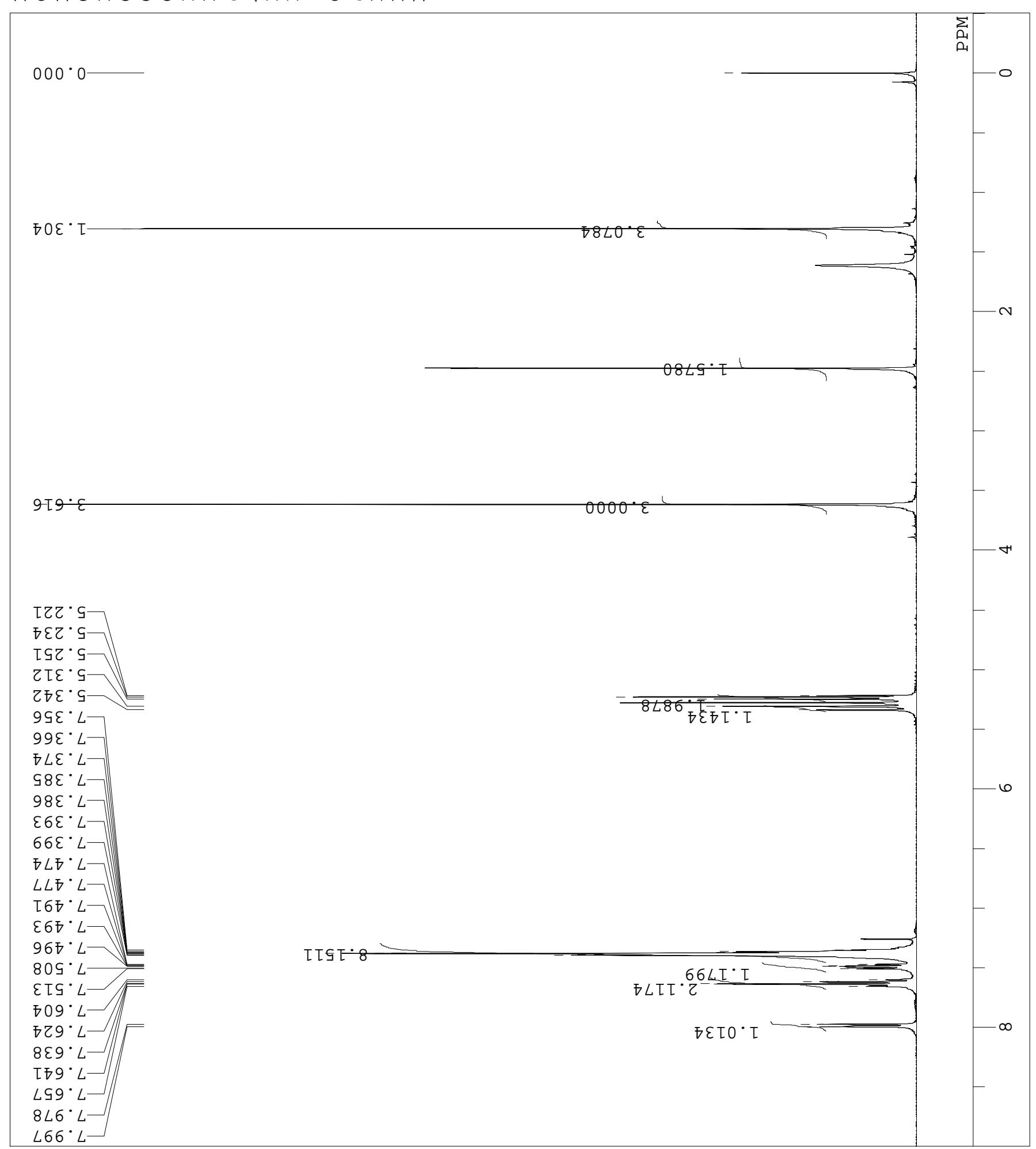

$9-\varepsilon$

$0000^{\circ}$
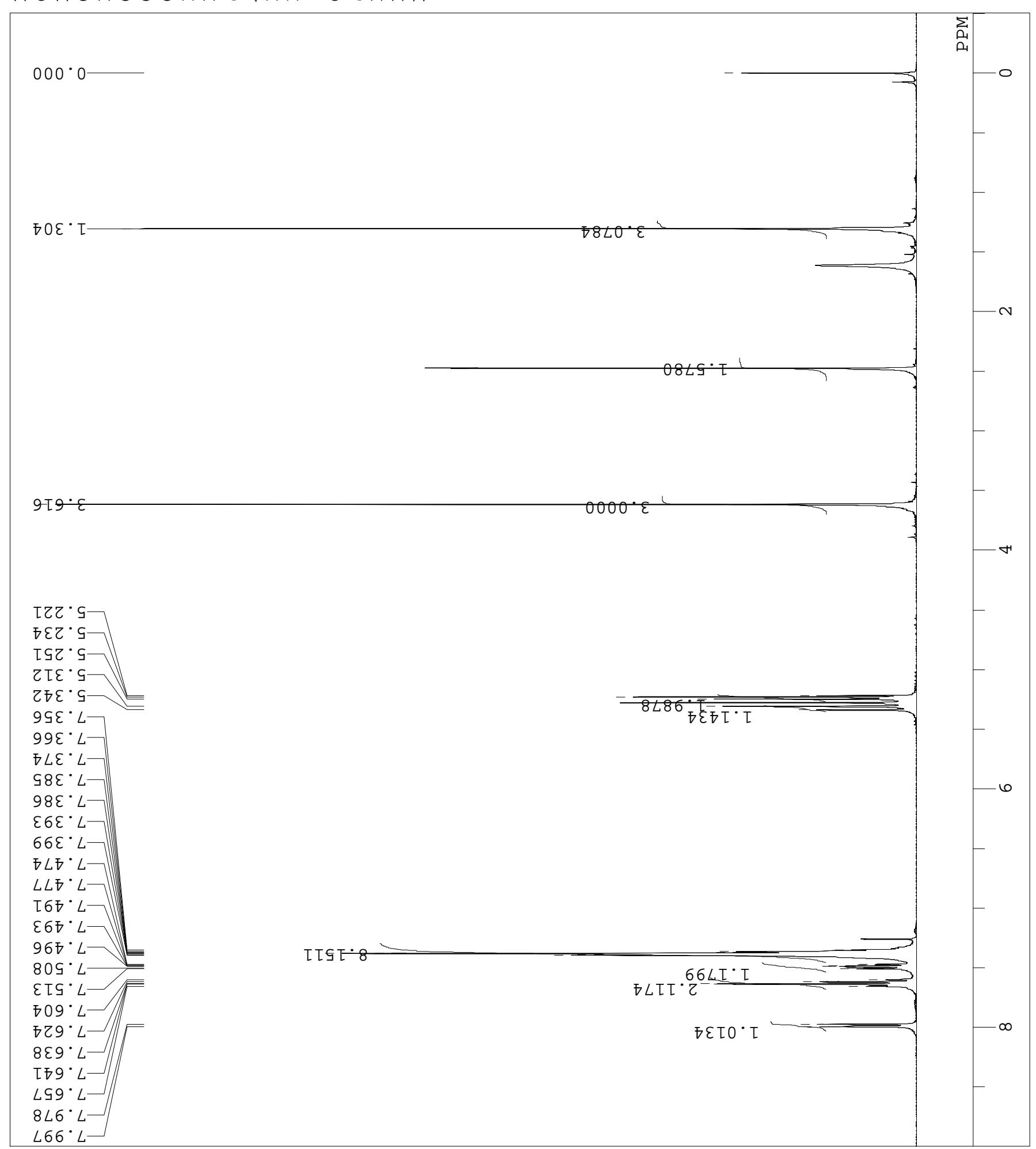

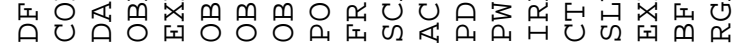


$\stackrel{\text { ก }}{\circ}$

ก)

$\ddot{\circ}$

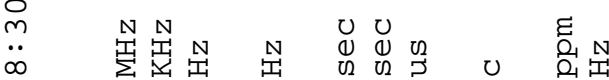

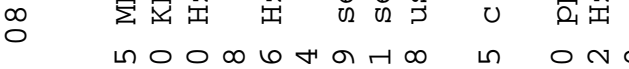

๙

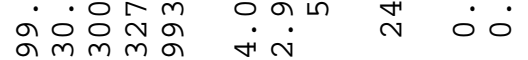

g $\quad m m m m a$ r
点昰

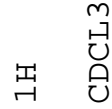

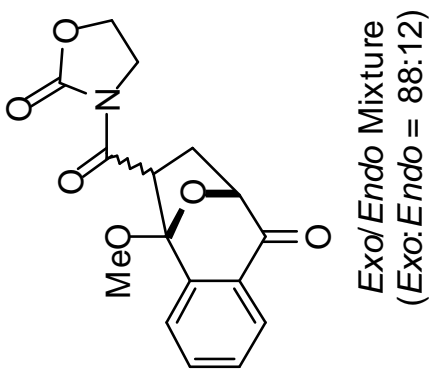

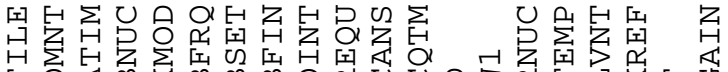

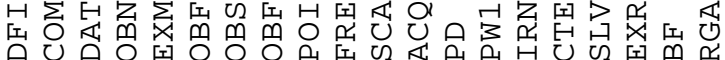

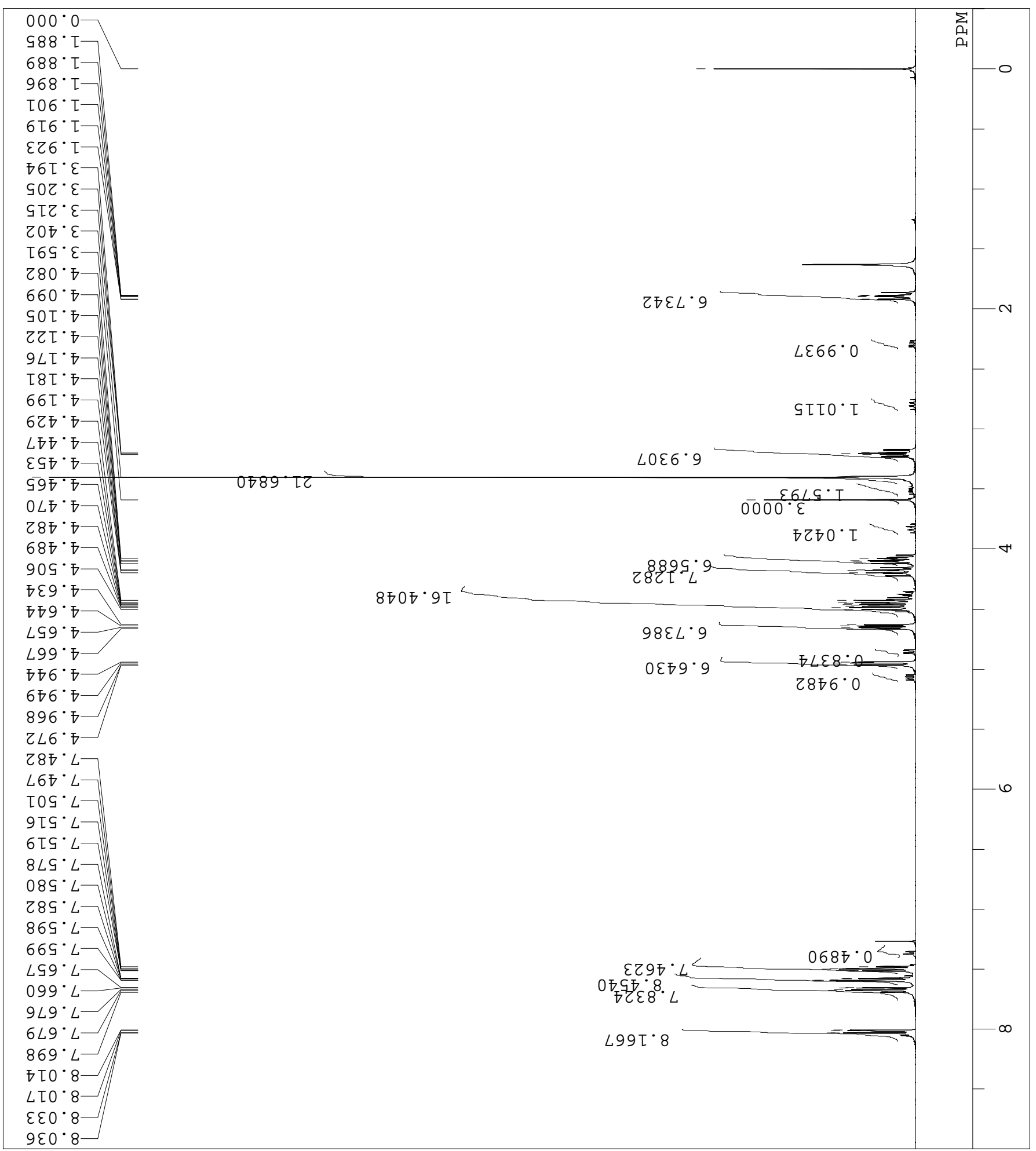


<smiles></smiles>

Endo-cycloadduct

\section{Racemate}

C-R8A CHROMATOPAC $\mathrm{CH}=1$ Report No. $=4$

Sample Name : KI-250-2

Sample ID :

Operator : KEI INOUE

Comment : CHIRALPAK AS, i-PrOH:HEXANE $=1: 19$, FLOW $=0.5 \mathrm{~mL} / \mathrm{min}, 35$ oC

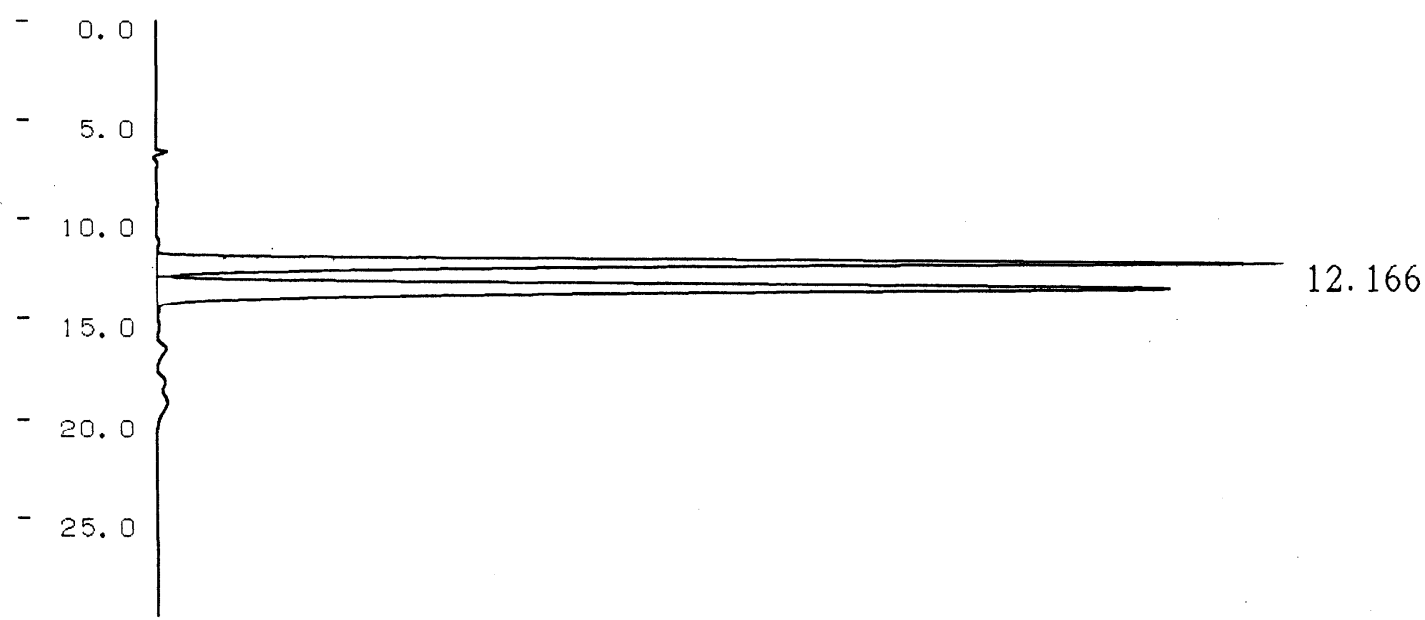

** CALCULATION REPORT **

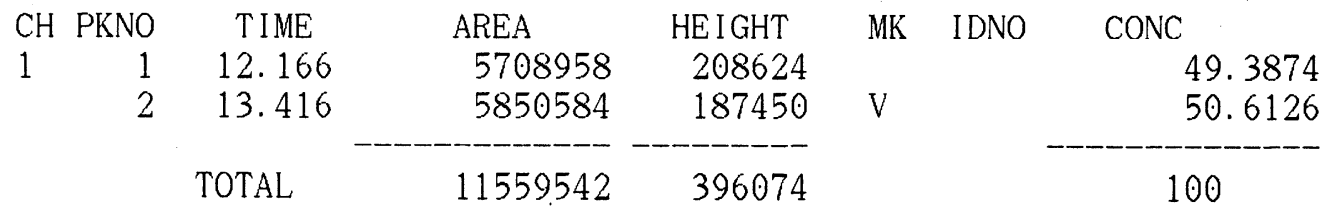

\section{$91 \%$ ee $(-)$}

C-R8A CHROMATOPAC $\mathrm{CH}=1$ Report No. $=4$

Sample Name: KI-284-2 (ENDO)

Sample ID :

Operator : KEI INOUE

Comment : CHIRALPAK AS, i-PrOH:HEXANE $=1: 19$, FLOW $=0.5 \mathrm{~mL} / \mathrm{min}, 35$ oC

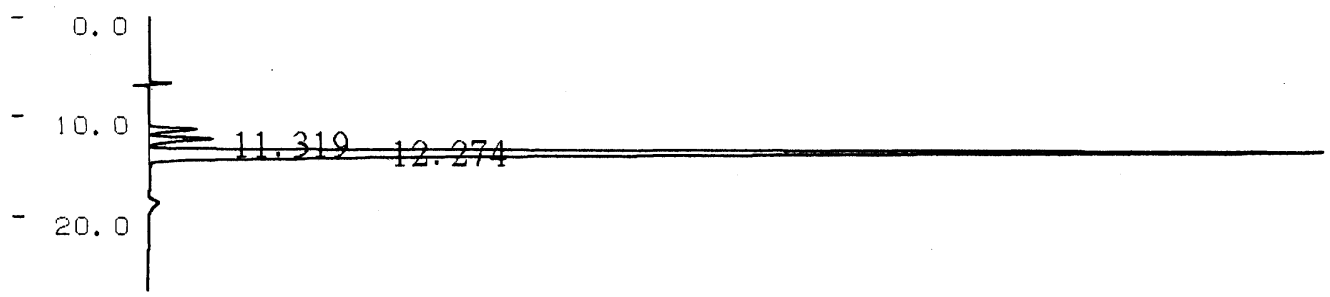

** CALCULATION REPORT **

\begin{tabular}{|c|c|c|c|c|c|c|}
\hline \multirow{5}{*}{$\begin{array}{lr}\mathrm{CH} & \text { PKNO } \\
1 & 1 \\
& 2 \\
& 3\end{array}$} & TIME & AREA & HE I GHT & MK & IDNO & CONC \\
\hline & 11.319 & 59593 & 2233 & & & 3.2711 \\
\hline & 12.274 & 80540 & 2971 & V & & 4.421 \\
\hline & 13.61 & 1681634 & 54619 & V & & 92.3079 \\
\hline & TOTAL & 1821766 & 59823 & & & 100 \\
\hline
\end{tabular}


<smiles>COC12OC(C(=O)c3ccccc31)C(C)(C(C)=O)O2</smiles>

Exo-cycloadduct

\section{Racemate}

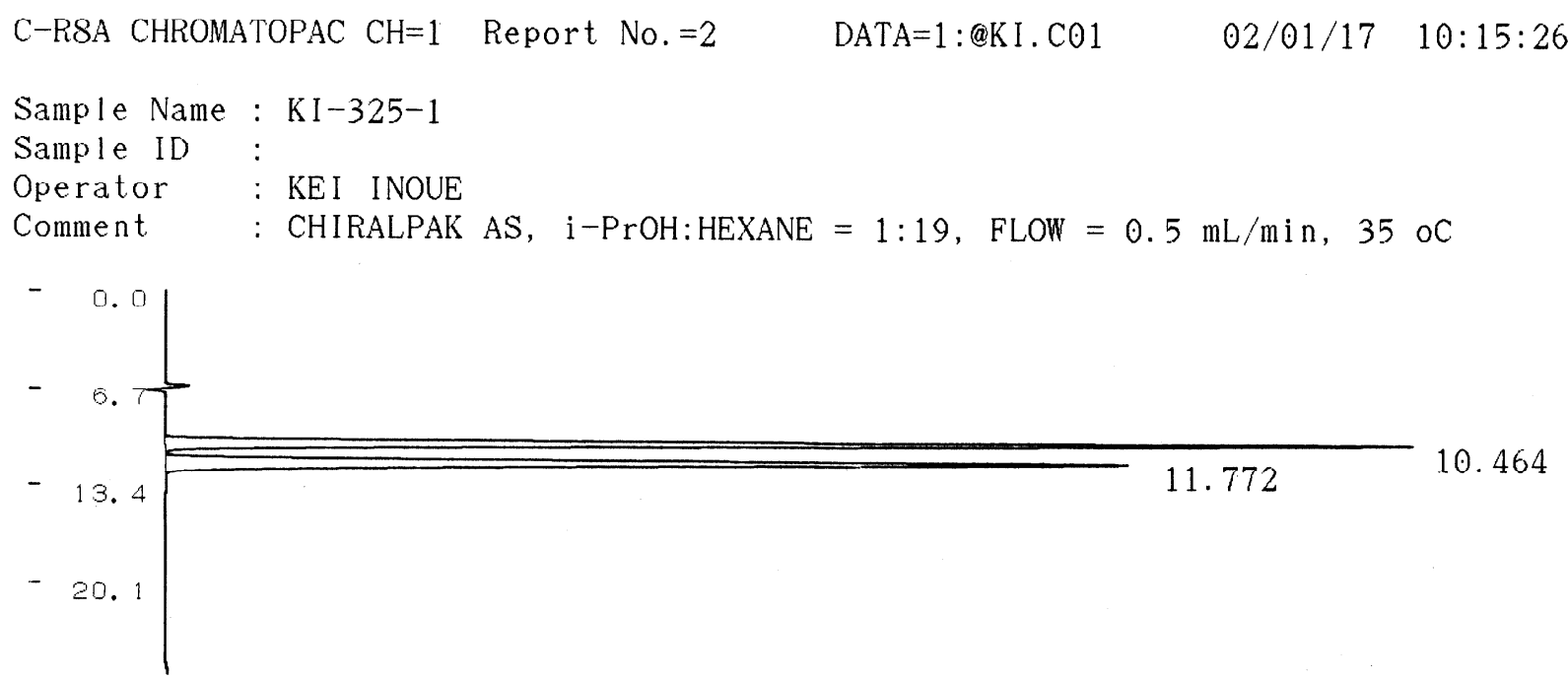

** CALCULATION REPORT

\begin{tabular}{rrrrrrrr} 
CH PKNO & TIME & \multicolumn{1}{c}{ AREA } & HEIGHT & MK & IDNO & \multicolumn{1}{c}{ CONC } & NAME \\
1 & 1 & 10.464 & 1200436 & 58697 & & 49.976 & \\
& 2 & 11.772 & 1201591 & 45209 & V & 50.024 & \\
& & & 2402027 & 103906 & & -100
\end{tabular}

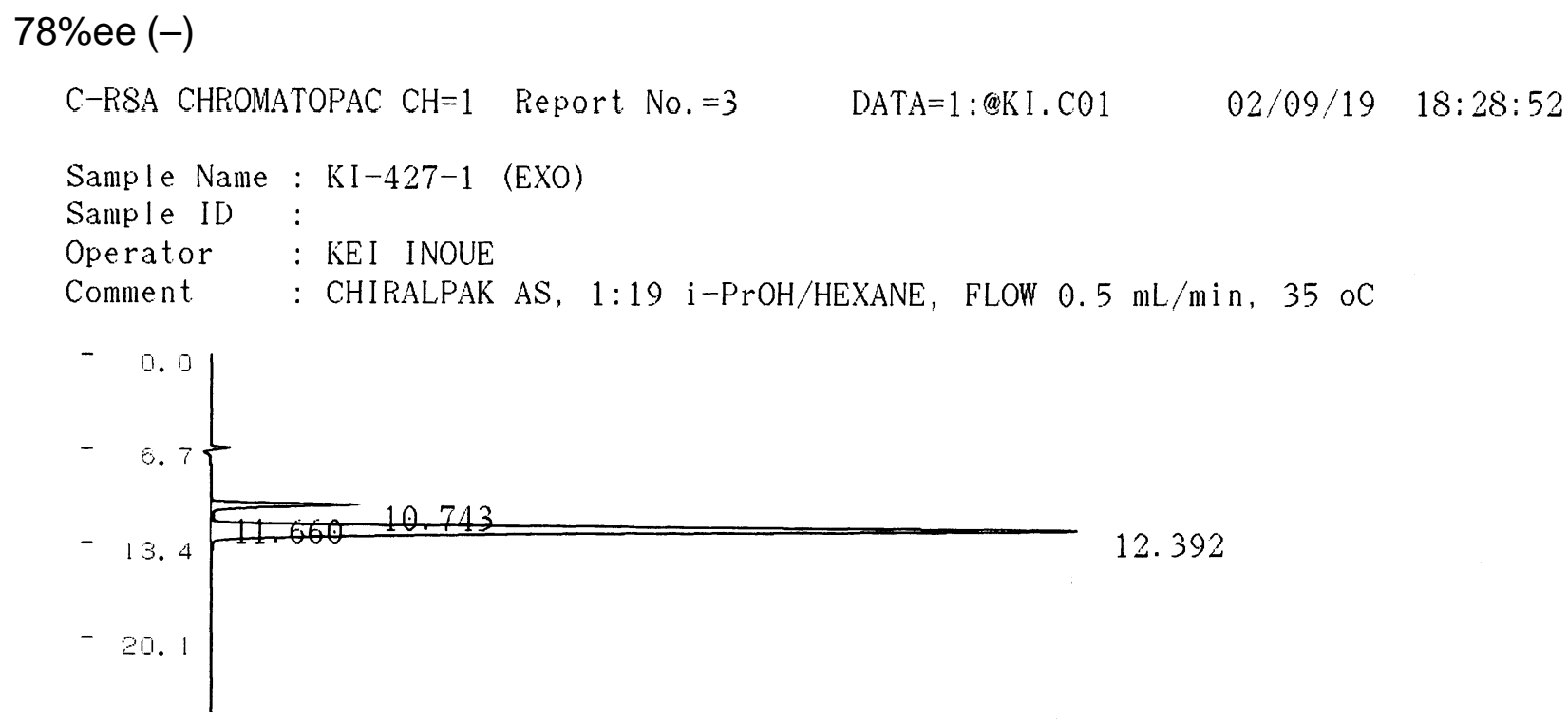

** CALCULATION REPORT **

\begin{tabular}{rrrrrrr} 
CH PKNO & TIME & \multicolumn{1}{c}{ AREA } & HEIGHT & MK & IDNO & \multicolumn{2}{c}{ CONC } \\
1 & 1 & 10.743 & 617281 & 29032 & & 10.9489 \\
& 2 & 11.66 & 11922 & 532 & $\mathrm{~V}$ & 0.2115 \\
& 3 & 12.392 & 5008635 & 169344 & SV & 88.8396 \\
& & & 5637838 & 198907 & & 100
\end{tabular}


<smiles>COC12OC(C(=O)OCc3ccccc3)C(OC1(C)OCc1ccccc1)C2=O</smiles>

Exo-cycloadduct

\section{Racemate}

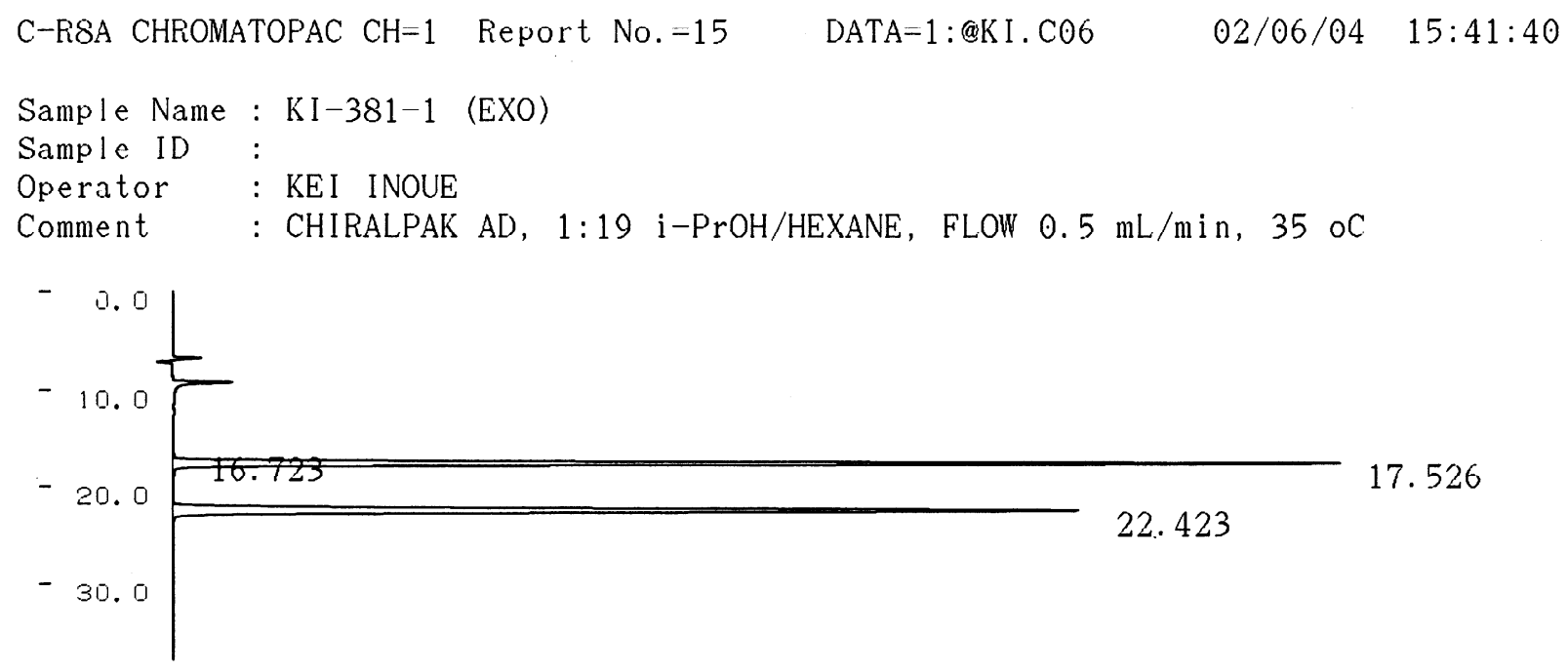

** CALCULATION REPORT **

\begin{tabular}{rrrrrrrr} 
CH PKNO & TIME & \multicolumn{1}{c}{ AREA } & HEIGHT & MK & IDNO & CONC & NAME \\
1 & 2 & 16.723 & 1236 & 69 & & 0.0491 & 50.0208 \\
& 3 & 17.526 & 1258183 & 55400 & $\mathrm{~V}$ & 49.93 \\
& 6 & 22.423 & 1255900 & 42996 & $\mathrm{~V}$ & \\
& & & TOTAL & 2515318 & 98465 & & ---100
\end{tabular}

\section{$87 \%$ ee (-)}

C-R8A CHROMATOPAC $\mathrm{CH}=1$ Report No $=2$

Sample Name : KI-429-1 (EXO)

Sample ID :

Operator : KEI INOUE

Comment : CHIRALPAK AD, $1: 19 \mathrm{i}-\mathrm{PrOH} / \mathrm{HEXANE}$, FLOW $0.5 \mathrm{~mL} / \mathrm{min}, 35 \mathrm{oC}$

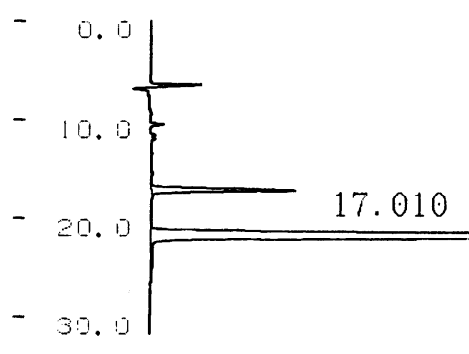

** CALCULATION REPORT *

\begin{tabular}{rrrrrrrr} 
CH PKNO & TIME & \multicolumn{1}{c}{ AREA } & HEIGHT & MK & IDNO & \multicolumn{1}{c}{ CONC } & NAME \\
1 & 1 & 17.01 & 298787 & 13369 & & 6.3406 & 93.6594 \\
& 2 & 21.631 & 4413471 & 153623 & $\mathrm{~S}$ & & -100
\end{tabular}


<smiles>COC12OC(CC1C(=O)N1CCOC1=O)C(=O)c1ccccc12</smiles>

\section{Exo-cycloadduct}

\section{Racemate}

C-R8A CHROMATOPAC $\mathrm{CH}=1$ Report No. $=4$

Sample Name : KI-348-1

Sample ID :

Operator : KEI INOUE

Comment : CHIRALPAK AS, $1: 4 \mathrm{i}$-PrOH/HEXANE, FLOW $0.5 \mathrm{~mL} / \mathrm{min}, 35$ oC

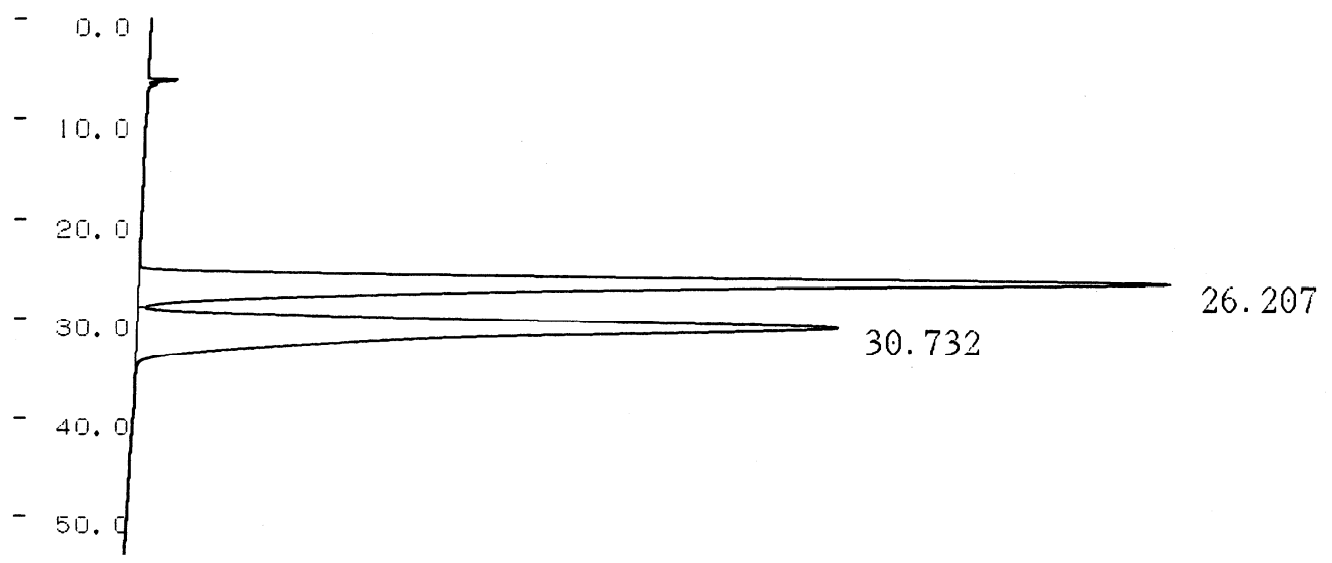

** CALCULATION REPORT **

\begin{tabular}{rrrrrrr} 
CH PKNO & TIME & \multicolumn{1}{c}{ AREA } & HEIGHT & MK & IDNO & \multicolumn{1}{c}{ CONC } \\
1 & 1 & 26.207 & 1006007 & 11958 & & 49.9888 \\
& 2 & 30.732 & 1006458 & 8119 & $\mathrm{~V}$ & 50.0112 \\
& & & & &
\end{tabular}

NAME

$98 \%$ ee $(+)$

C-R8A CHROMATOPAC $\mathrm{CH}=1$ Report No. $=3$

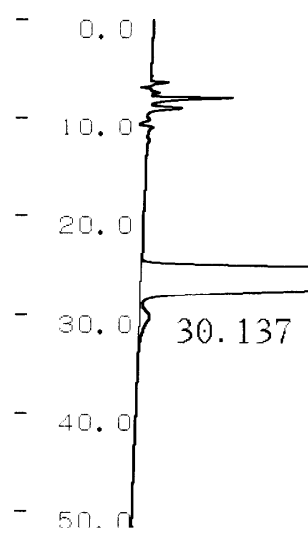

** CALCULATION REPORT **

\begin{tabular}{rrrrrrrr} 
CH PKNO & TIME & \multicolumn{1}{c}{ AREA } & HE IGHT & MK & IDNO & \multicolumn{1}{c}{ CONC } & NAME \\
1 & 1 & 25.897 & 2532798 & 32144 & & 98.9632 & 1.0367 \\
& 2 & 30.137 & 26534 & 232 & $V$ & & V \\
& & & TOTAL & 2559331 & 32376 & & -100
\end{tabular}

